\title{
Test-Retest Reliability of Short-Interval Intracortical Inhibition Assessed by Threshold-Tracking and Automated Conventional Techniques
}

\author{
(DChristina S.-Z. Nielsen, ${ }^{1,2}$ Gintaute Samusyte, ${ }^{3}$ Kirsten Pugdahl, ${ }^{1,2}$ (D)Jakob U. Blicher, ${ }^{4}$ \\ Anders Fuglsang-Frederiksen, ${ }^{1,2}$ Bülent Cengiz, ${ }^{5}$ and Hatice Tankisi ${ }^{1,2}$
}

https://doi.org/10.1523/ENEURO.0103-21.2021

${ }^{1}$ Department of Clinical Neurophysiology, Aarhus University Hospital, Aarhus 8200, Denmark, ${ }^{2}$ Department of Clinical Medicine, Faculty of Health, Aarhus University, Aarhus 8200, Denmark, ${ }^{3}$ Department of Neurology, Medical Academy, Lithuanian University of Health Sciences, Kaunas 44307, Lithuania, ${ }^{4}$ Center of Functionally Integrative Neuroscience, Department of Clinical Medicine, Aarhus University, Aarhus 8200, Denmark, and ${ }^{5}$ Department of Neurology, Gazi University, Faculty of Medicine, Ankara 06500, Turkey

\begin{abstract}
Two novel short-interval intracortical inhibition (SICl) protocols, assessing $\mathrm{SICl}$ across a range of interstimulus intervals (ISIs) using either parallel threshold-tracking transcranial magnetic stimulation (TT-TMS) or automated conventional TMS (cTMS), were recently introduced. However, the test-retest reliability of these protocols has not been investigated, which is important if they are to be introduced in the clinic. SICl was recorded in 18 healthy subjects using TT-TMS (T-SICl) and cTMS (A-SICl). All subjects were examined at four identical sessions, i.e., morning and afternoon sessions on $2 \mathrm{~d}, 5-7 \mathrm{~d}$ apart. Both $\mathrm{SICl}$ protocols were performed twice at each session by the same observer. In one of the sessions, another observer performed additional examinations. Neither intraobserver nor interobserver measures of SICl differed significantly between examinations, except for T-SICI at ISI $3 \mathrm{~ms}(p=0.00035)$ and A-SICI at ISI $2.5 \mathrm{~ms}(p=0.0103)$. Intraday reliability was poor-togood for $\mathrm{A}-\mathrm{SICl}$ and moderate-to-good for T-SICl. Interday and interobserver reliabilities of T-SICI and A-SICI were moderate-to-good. Although between-subject variation constituted most of the total variation, $\mathrm{SICl}$ repeatability in an individual subject was poor. The two $\mathrm{SICl}$ protocols showed no considerable systematic bias across sessions and had a comparable test-retest reliability profile. Findings from the present study suggest that both $\mathrm{SICl}$ protocols may be reliably and reproducibly employed in research studies, but should be used with caution for individual decision-making in clinical settings. Studies exploring reliability in patient cohorts are warranted to investigate the clinical utility of these two $\mathrm{SICl}$ protocols.
\end{abstract}

Key words: automated conventional TMS; short-interval intracortical inhibition; test-retest reliability; thresholdtracking TMS; transcranial magnetic stimulation

\section{Significance Statement}

Threshold-tracking short-interval intracortical inhibition (T-SICI) measured with threshold-tracking transcranial magnetic stimulation (TT-TMS) was introduced two decades ago (Fisher et al., 2002). Earlier studies have shown that T-SICI may be used for diagnosing ALS (Vucic and Kiernan, 2006, 2008). However, limitations of the existing serial T-SICI protocol was recently reported and two novel SICI protocols, with potential experimental and clinical utility, were introduced (Samusyte et al., 2018; Tankisi et al., 2021a). These studies were conducted on healthy subjects. The test-retest reliability of the aforementioned protocols was investigated over four identical sessions on 2 days, 5-7 days apart. These results suggest that the two SICl protocols may be reliably and reproducibly employed in research studies with healthy subjects. 


\section{Introduction}

Conventional transcranial magnetic stimulation (cTMS) uses magnetic stimulation to measure cortical excitability by applying constant stimulus intensities. If stimulation is applied over the motor cortex, a motor evoked potential (MEP) can be recorded (Kujirai et al., 1993). Cortical excitability can then be measured as changes in averaged MEP (Kujirai et al., 1993).

Threshold-tracking TMS (TT-TMS) is an unconventional TMS method, which also measures cortical excitability (Fisher et al., 2002). Contrary to cTMS, the MEP amplitude is predefined and kept constant by adjusting the stimulus intensities, thus enabling continuous tracking of the motor thresholds and allowing for fluctuations in cortical excitability (Fisher et al., 2002). The method was introduced to counteract restrictions in cortical excitability fluctuations and MEP variability (Fisher et al., 2002; Groppa et al., 2012).

Short-interval intracortical inhibition (SICl) measures cortical inhibition and is a TMS protocol in which two stimuli are delivered with an interstimulus interval (ISI) of 1-7 ms (Kujirai et al., 1993). The first subthreshold stimulus (conditioning stimulus, CS) is followed by a second suprathreshold stimulus (test stimulus; Kujirai et al., 1993), which in TT-TMS is continuously adjusted based on the recorded MEP amplitude (Fisher et al., 2002). In conventional amplitude $\mathrm{SICI}(\mathrm{A}-\mathrm{SICl})$, cortical inhibition is measured as the relative change in MEP amplitude (Kujirai et al., 1993). In T-SICI (SICI measured by TT-TMS), cortical inhibition is measured as the relative change in stimulus intensity (Fisher et al., 2002).

The precise physiological mechanisms behind $\mathrm{SICl}$ are unknown, but $\mathrm{SICl}$ at an ISI of $1 \mathrm{~ms}\left(\mathrm{SICl}_{1 \mathrm{~ms}}\right)$ is thought to reflect neuronal refractoriness or extrasynaptic GABA-A signaling (Fisher et al., 2002; Roshan et al., 2003; Stagg et al., 2011), whereas $\mathrm{SICl}$ at an ISI of $2.5 \mathrm{~ms}\left(\mathrm{SICl}_{2.5 \mathrm{~ms}}\right)$, and an ISI of $3 \mathrm{~ms}\left(\mathrm{SICl}_{3 \mathrm{~ms}}\right)$ are thought to reflect synaptic GABA-Aergic inhibition (Ziemann et al., 1996; Vucic and Kiernan, 2006). Earlier studies have shown that T-SICI

Received March 8, 2021; accepted August 20, 2021; First published September 24, 2021.

The authors declare no competing financial interests.

Author contributions: C.S.-Z.N., K.P., J.U.B., A.F.-F., B.C., and H.T. designed research; C.S.-Z.N. and H.T. performed research; C.S.-Z.N. and G. S. analyzed data; C.S.-Z.N., G.S., K.P., J.U.B., A.F.-F., B.C., and H.T. wrote the paper.

This work was supported by the Lundbeck Foundation, A.P. Møller Fonden (Fonden til Lægevidenskabens Fremme Grant 17-L-0365), the Aage \& Johanne Louis-Hansens Foundation Grant 18-2B-2454, and the Independent Research Fund Denmark Grant DFF 7025-00066.

K. Pugdahl's present address: Department of Clinical Medicine, Occupational Medicine, Aarhus University Hospital, Aarhus 8200, Denmark.

Acknowledgements: We thank Professor H. Bostock (Department of Neuromuscular Diseases, University College London Queen Square Institute of Neurology, London, United Kingdom) for his development and consultation on the TMS protocols used.

Correspondence should be addressed to Hatice Tankisi at hatitank@rm.dk.

https://doi.org/10.1523/ENEURO.0103-21.2021

Copyright $@ 2021$ Nielsen et al.

This is an open-access article distributed under the terms of the Creative Commons Attribution 4.0 International license, which permits unrestricted use, distribution and reproduction in any medium provided that the original work is properly attributed. may be used for diagnosing ALS and has been suggested as a biomarker (Vucic and Kiernan, 2006, 2008; Menon et al., 2015; Vucic and Rutkove, 2018). These studies applied a protocol of serial tracking that estimated T-SICl at successively increasing ISIs (Vucic and Kiernan, 2006; Vucic et al., 2006; Matamala et al., 2018). A slightly different tracking strategy was applied in a recent study, in which comparability and reliability of $\mathrm{T}-\mathrm{SICl}$ and automated A-SICl were explored at ISI $2.5 \mathrm{~ms}$ at four different CS intensities in healthy subjects (Samusyte et al., 2018). The CS intensities were tracked in parallel in a pseudorandomized order, a commonly used approach in cTMS (Samusyte et al., 2018). A good correlation of SICl obtained by the two techniques was found across the whole range of CS (Samusyte et al., 2018).

More recently, a good correlation of automated $\mathrm{A}-\mathrm{SICl}$ and T-SICl at a single CS intensity and ISIs of 1-7 ms with a parallel tracking strategy and important limitations of serial tracking were demonstrated in healthy subjects (Tankisi et al., 2021a). It was proposed that because of a smaller between-subject variability among healthy individuals, A-SICI may be better at demonstrating a pathologic loss of inhibition, which has been observed as an early feature of motor neuron disease (Vucic and Kiernan, 2006). A recent study has demonstrated that both techniques performed well at discriminating ALS patients from patient controls with $\mathrm{T}-\mathrm{SICl}$ being most reduced before the upper motor neuron signs become apparent (Tankisi et al., 2021b). However, none of the studies assessed the test-retest reliability of the methods, which is important if an investigation is to be used for diagnostic purposes or interventional studies. A trend for improved reproducibility of T-SICl $2.5 m s$ has been reported (Samusyte et al., 2018), but it remains unclear whether this applies to other SICI ISIs. Further studies are needed to explore the utility of T$\mathrm{SICl}$ and $\mathrm{A}-\mathrm{SICl}$ in diagnostic decision-making in ALS, but the comparison of these two methods' reproducibility and reliability in healthy subjects should be investigated before implementation in clinics. Moreover, T$\mathrm{SICl}$ and $\mathrm{A}-\mathrm{SICl}$ may potentially investigate different motor neuron pools (Samusyte et al., 2018), and may therefore supplement each other in diagnostics and intervention studies. Therefore, the present study aimed to explore the repeatability and observer reproducibility of the two novel automated conventional and thresholdtracking $\mathrm{SICl}$ protocols across ISIs $1-7 \mathrm{~ms}$ in healthy subjects. The study also aimed to assess intraday and interday reliability in relation to diurnal variations, which may affect the reliability of $\mathrm{SICl}$ measurements (Matamala et al., 2018). No previous study has examined these parameters of T$\mathrm{SICl}$ parallel and automated $\mathrm{A}-\mathrm{SICl}$ on such an extensive scale.

\section{Materials and Methods}

\section{Subjects}

The study was conducted at Department of Clinical Neurophysiology, Aarhus University Hospital, Denmark, from February 2018 to August 2018. Inclusion criteria were: age above 18 years; absence of neurologic or 


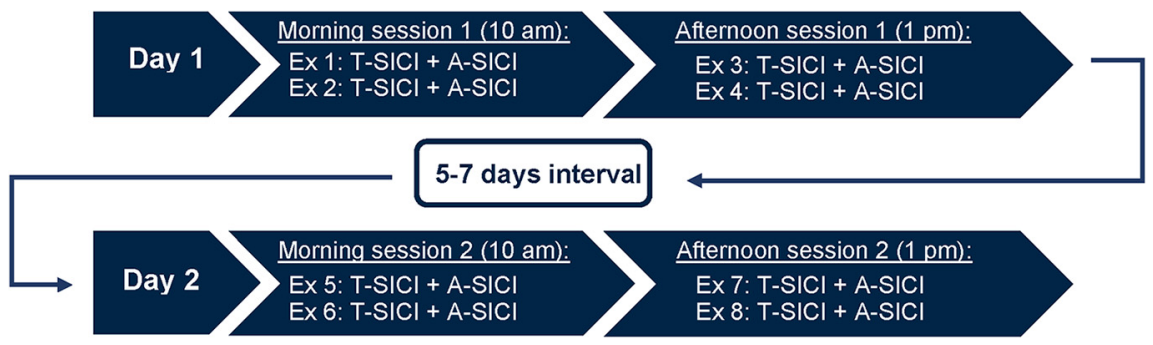

Figure 1. Study design. Each subject was examined on four sessions on two separate days, 5-7 d apart: two sessions in the mornings and two sessions in the afternoons. A-SICI and T-SICl protocols were repeated twice in each session. These examinations were performed by the same observer (observer 1). Two TMS examinations (one A-SICI examination and one T-SICI examination) were additionally performed by a second observer (observer 2) on each subject. The examinations were done in continuation of one of the sessions. In total, nine A-SICl and nine T-SICl examinations were performed on each subject.

psychiatric disorders. Exclusion criteria were: pregnancy; use of medication known to affect the nervous system; metal implants. All participating subjects were screened using a modified TMS safety questionnaire (Rossi et al., 2011) and a gross neurologic examination.

Twenty healthy subjects (nine females) were recruited. One subject (S5) was excluded because of undetectable MEP, another subject (S7) because of inability to relax the hand muscles. Eighteen subjects (eight females, mean age: 56.9 years, SD: 12.3 ; range: $41-77$ years) were recruited. One subject (S4, male, age: 46 years) completed all nine TT-TMS examinations, but only seven cTMS examinations by observer 1 because of time restraint. The subject was excluded from the analysis of reliability of cTMS data.

Written informed consent was obtained from all subjects in accordance with the Declaration of Helsinki II. The project was approved by The Central Denmark Region Committees on Health Research Ethics (case: 1-10-72201-17) and the Danish Data Protection Agency.

\section{Study design}

To investigate intraobserver test-retest reliability, each subject was investigated by the same observer (observer 1) on two separate days, 5-7 d apart. On the first examination day, two sessions were conducted: a morning session (10.00-11.30 A.M.) and an afternoon session (12:30 P.M.). On the second examination day, a morning session (10-11:30 A.M.) and an afternoon session (12:30 P.M.) were conducted again (Fig. 1). Each session consisted of four TMS examinations: two A-SICl examinations and two $\mathrm{T}-\mathrm{SICl}$ examinations. On each examination day, each subject underwent four A-SICl examinations and four $\mathrm{T}-\mathrm{SICl}$ examinations. Thus, eight $\mathrm{A}-\mathrm{SICl}$ examinations and eight $\mathrm{T}-\mathrm{SICl}$ examinations were conducted on each subject in total by observer 1 (Fig. 1).

All examinations were executed at the same time of day for each subject. Each examination lasted on average $15 \mathrm{~min}$, giving approximately $1 \mathrm{~h}$ in total for each session.

To investigate interobserver reliability and reproducibility, a second observer (observer 2) performed an additional TTTMS and cTMS examination on each subject in continuation of one of the recording sessions. Interobserver (observer 2) examinations were measured either at the morning session of day $1(n=3)$, the afternoon session of day $1(n=7)$, the morning session of day $2(n=4)$ or the afternoon session of day $2(n=4)$ because of practical limitations.

Subjects were instructed to restrain from coffee $(12 \mathrm{~h})$, alcohol (24 h), and exhaustive exercise (48 h) before TMS examination.

\section{Experimental setup}

The subjects were comfortably seated during the examinations, with their right arm resting in a relaxed position on a pillow placed on their lap. The subjects were instructed to stay relaxed but vigilant. MEP responses were recorded from the relaxed right first dorsal interosseous (FDI) muscle of the right hand using Ag/AgCl ECG electrodes (Ambu WhiteSensor 40713). The active electrode was placed on the belly of the FDI muscle, the reference electrode on the second metacarpophalangeal joint. The ground electrode was placed on the dorsum of the hand. Skin temperature of the subject's right hand was measured before and after each examination, and a constant temperature was ensured with a heating lamp throughout the examination.

The EMG signal was amplified ( $1000 \times$ gain) and filtered (3$3000 \mathrm{~Hz}$ ) using a two-channel isolated amplifier (D440-2, Digitimer Ltd.). To remove $50 / 60-\mathrm{Hz}$ noise, Humbug Noise Eliminator (Digitimer Ltd.) was used as well as a 2000 VA medical transformer (IMEDe 2000, Noratel). To digitize amplified signals, a NI USB-6251 data acquisition system (eight inputs, 16-bit, 1.25 MS/s, National Instruments) was used.

\section{TMS}

Cortical function was assessed using a 70-mm figureof-eight coil (D70 Remote Coil, reference number 319000) connected to two Magstim $200^{2}$ stimulators in Bistim mode (Magstim Co Ltd). Posterior-anterior current flow in the subject's motor cortex was induced by placing the coil over the left hemisphere with the coil handle angled $45^{\circ}$ postero-laterally to the midsagittal line.

The hand motor hotspot was located by moving the coil in anterior-posterior and medial-lateral directions to induce a MEP of $0.2 \mathrm{mV}$ using a minimal stimulus intensity. Once located, coil-positioning over the hand motor hotspot was kept constant by drawing the coil outline onto a swimming cap worn by the subject. This procedure was 


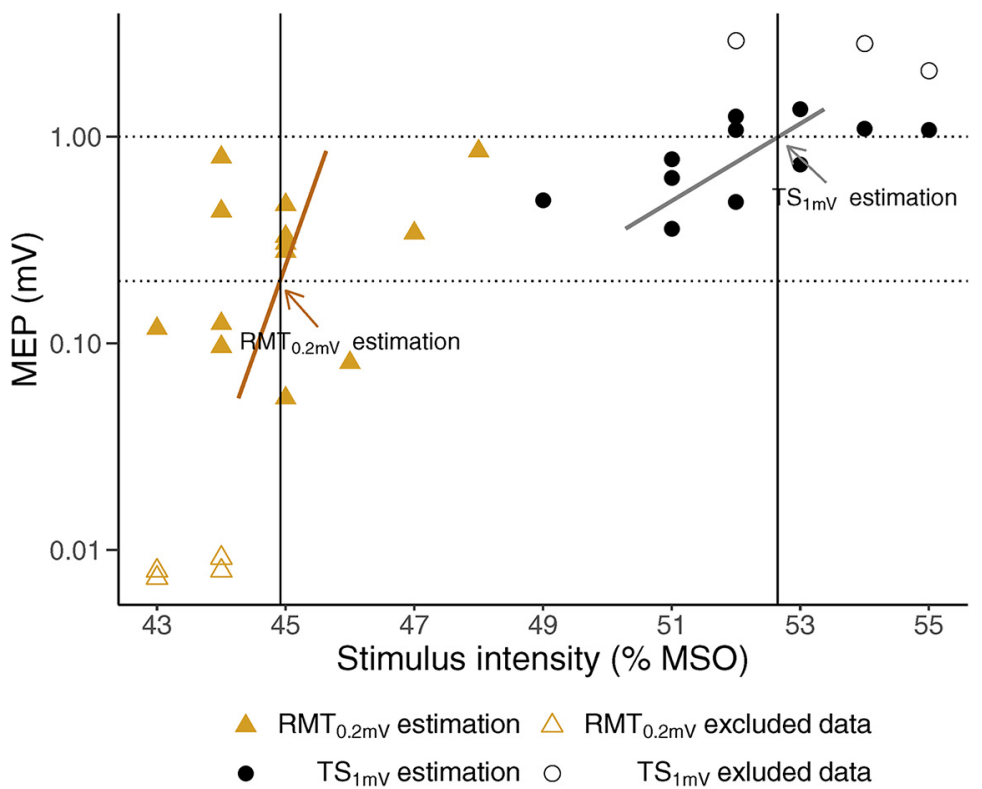

Figure 2. Estimation of $\mathrm{RMT}_{0.2 \mathrm{mv}}$ and $\mathrm{TS}_{1 \mathrm{mv}}$. Scatter plots of recorded MEP against stimulus intensity (in \% MSO). RMT $0.2 \mathrm{mv}$ is the test stimulus (in \% MSO) required to evoke a peak-to-peak MEP of $0.2 \mathrm{mV}$, whereas $\mathrm{TS}_{1 \mathrm{mV}}$ is the test stimulus (in \% MSO) required to evoke a peak-to-peak MEP of $1 \mathrm{mV}$. The figure conceptually shows how RMT $0.2 \mathrm{mv}$ and $\mathrm{TS}_{1 \mathrm{mv}}$ are estimated. The MEPs were recorded during the $\mathrm{RMT}_{0.2 \mathrm{mv}}$ (orange triangle) and $\mathrm{TS}_{1 \mathrm{mV}}$ (black circle) estimation, before initiation of $\mathrm{SICl}_{\mathrm{Croto}} \mathrm{pols}_{\mathrm{RMT}} \mathrm{R}_{0.2 \mathrm{mv}}$ and $\mathrm{TS}_{1 \mathrm{mV}}$ estimation (solid black vertical lines) were calculated from the intersect of target MEP (dotted horizontal lines) and weighted semi-logarithmic regressions (orange and gray lines), shown by arrows in the figure. Low $(<0.02 \mathrm{mV})$ or high ( $>2 \mathrm{mV}) \mathrm{MEP}$ responses (open circles and open triangles) were excluded from the calculation.

repeated before each examination. A spring balancer (SiraFlex, type B) helped steadying the coil. Stimulation frequency was $0.2 \mathrm{~Hz}$.

Automated stimulator control, stimulus delivery, data acquisition and calculation of TMS parameters were managed by the computer software QTRACW (Institute of Neurology, University College London, United Kingdom, distributed by Digitimer Ltd.) using bespoke recording protocols (QTMS-2017).

\section{Resting motor threshold (RMT)}

RMT estimates cortical excitability by measuring the lowest stimulation intensity required to elicit a predefined target MEP. The RMT is estimated before initiation of the $\mathrm{SICl}$ protocol and is used as a baseline for calculating CS intensities in SICl. In TT-TMS, the RMT is continuously estimated, "tracked," during the paired-pulse protocol, as opposed to automated cTMS.

After localization of the motor hotspot, but before SICI protocol initiation, the lowest stimulus intensities (measured in percentage of maximum stimulator output; \% MSO) required for eliciting a peak-to-peak target MEP of $0.2 \mathrm{mV}$ $\left(\mathrm{RMT}_{0.2 \mathrm{mV}}\right)$ and a peak-to-peak target MEP of $1 \mathrm{mV}\left(\mathrm{TS}_{1 \mathrm{mV}}\right)$ were estimated by threshold-tracking (Fig. 2). The size of the MEPs was analyzed online by QTRACW, which then automatically adjusted the Magstim $200^{2}$ stimulator output. A proportional tracking mode with a maximum step of $2 \%$ MSO was used: the stimulation intensities were adjusted depending on the percentage error of a single MEP (decreased, increased or unchanged if the MEP was above, below, or on target, respectively). A 20\% tracking error (on a logarithmic scale) was allowed, and the threshold estimate was considered valid if the MEP hit or bracketed the target line. The $\mathrm{RMT}_{0.2 \mathrm{mv}}$ and $\mathrm{TS}_{1 \mathrm{mv}}$ tracking was deemed stable when six valid estimates had been obtained. $\mathrm{RMT}_{0.2 \mathrm{mv}}$ and $\mathrm{TS}_{1 \mathrm{mv}}$ were automatically calculated by applying a weighted logarithmic regression (Fig. 2). This approach is based on work by Fisher et al. (2002), who found that the stimulus-response curve between the stimulus intensity for single pulse TMS and the MEP amplitude was approximately exponential over a 100-fold range of responses. When plotted on a logarithmic scale, the relationship between stimulus intensity and MEP amplitude was approximately linear in the interval from 0.02 to $2 \mathrm{mV}$. Target MEP was set at $0.2 \mathrm{mV}$, the midpoint in this log-linear interval (Fisher et al., 2002). Furthermore, the MEP distributions are often skewed (Nielsen, 1996), and the variability in MEP size, which could represent excitability fluctuations in cortical pyramidal cells and spinal motor neurons, may differ across stimulus intensities (Kiers et al., 1993). Thus, logarithmic transformation of amplitude data has been proposed to ensure normal distributions and to at least somewhat reduce the variability spread across the stimulus intensities (Nielsen, 1996; Goetz et al., 2014). Vucic et al. (2006) deduced that by setting a target MEP located in the midpoint of the log-linear interval, the variability in MEP amplitude translates to smaller changes in stimulus intensity, potentially overcoming these limitations.

The tracked $\mathrm{RMT}_{0.2 \mathrm{mv}}$ estimate was then used to set the CS for both $\mathrm{T}-\mathrm{SICl}$ and $\mathrm{A}-\mathrm{SICl}$ protocols to ensure a comparable intensity between the techniques. The $\mathrm{SICl}$ protocol was then initiated (Fig. 3). 

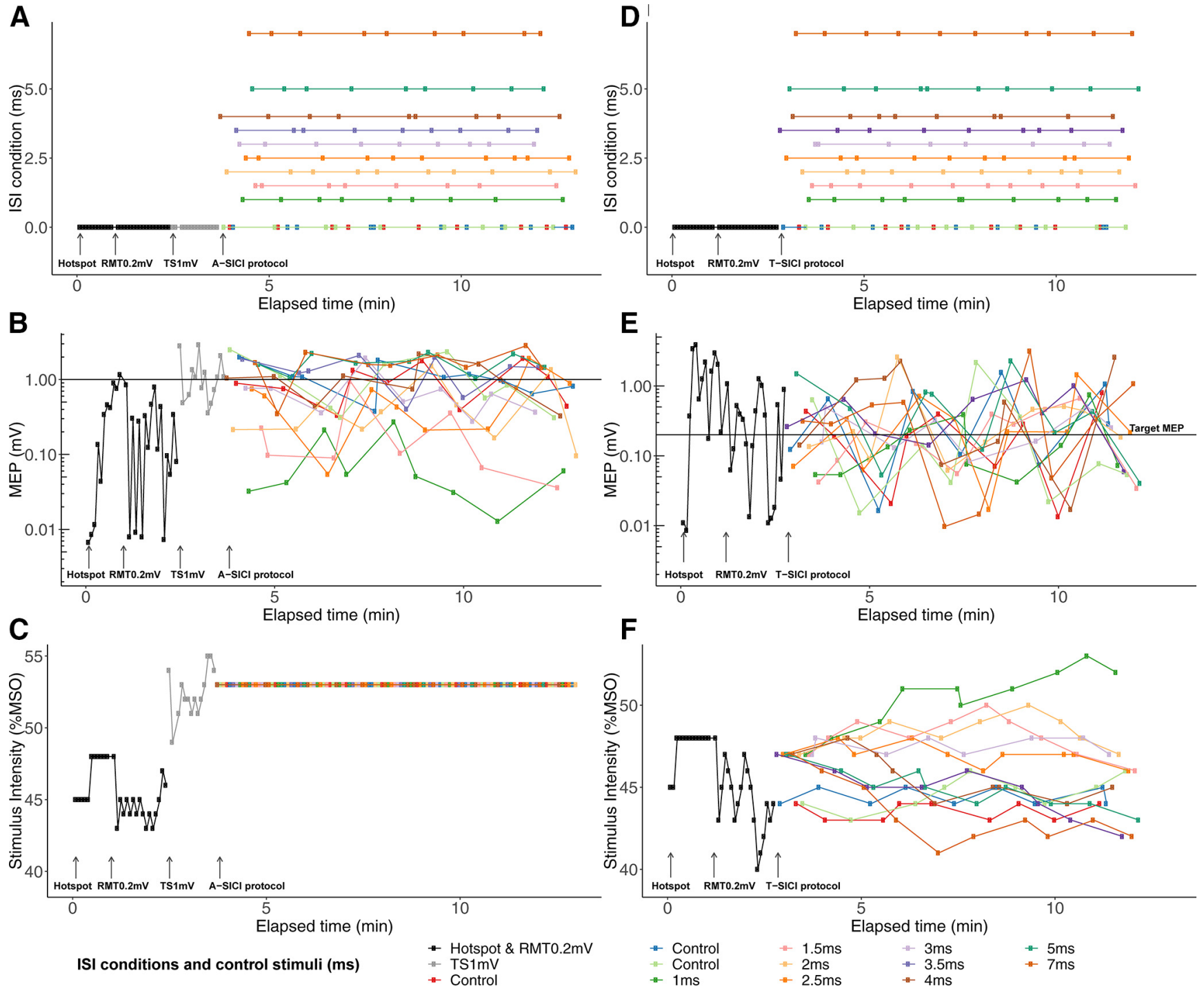

Figure 3. Example recording of $\mathrm{A}-\mathrm{SICl}$ and $\mathrm{T}-\mathrm{SICl}$. Example recording of $\mathrm{A}-\mathrm{SICl}$ recorded with $\mathrm{cTMS}(\boldsymbol{A}-\boldsymbol{C})$ and $\mathrm{T}-\mathrm{SICl}$ recorded with TT-TMS $(\boldsymbol{D}-\boldsymbol{F})$ for subject 1 of the first examination in morning session 1 from QTRACW. Each color depicts either a different ISI condition (ISI $=1-7 \mathrm{~ms}$ ) or a control stimulus $(\mathrm{ISI}=0 \mathrm{~ms})$. After location of the motor hotspot, $\mathrm{RMT}_{0.2 \mathrm{mv}}$ and $\mathrm{TS}_{1 \mathrm{mV}} \mathrm{Were}$ estimated, and the A-SICI protocol was initiated $(\boldsymbol{A}-\boldsymbol{C})$. Likewise, after $\mathrm{RMT}_{0.2 \mathrm{mv}}$ estimation, the T-SICl protocol was initiated $(\boldsymbol{D}-\boldsymbol{F})$. For $\mathrm{T}-\mathrm{SICI}$, the nine different conditions and control stimuli $(\boldsymbol{D})$ were pseudorandomized and tracked in parallel $(\boldsymbol{E})$. Paired stimulus intensities were adjusted continuously to obtain a target MEP of $0.2 \mathrm{mV}(\boldsymbol{F})$. Likewise, for A-SICl nine different conditions and control stimuli $(\boldsymbol{A})$ were pseudorandomized $(\boldsymbol{B})$. However, the paired stimulus intensities were kept constant for $\mathrm{A}-\mathrm{SICl}(\boldsymbol{C})$.

\section{T-SICI protocol}

The T-SICl protocol was initiated after $\mathrm{RMT}_{0.2 \mathrm{mv}}$ estimation and was executed by QTRACW. The conditioning and test stimulus pairs were given at nine different conditions, with each condition comprising of a different ISI. The ISIs were 1, 1.5, 2, 2.5, 3, 3.5, 4, 5, and $7 \mathrm{~ms}$ (Fig. 3D$F)$. For adequate monitoring of the control condition, $\mathrm{RMT}_{0.2 \mathrm{mv}}$ was continuously tracked on three independent channels using $1 \%$ MSO steps. The control and paired stimuli were delivered in a pseudorandomized order (Fig. $3 D$ ). The protocol stopped after each of the nine ISI conditions had been delivered ten times (giving 90 paired stimuli), and $\mathrm{RMT}_{0.2 \mathrm{mv}}$ estimation had been conducted 30 times (giving 30 single stimuli).
The CS and the test stimulus were adjusted continuously. The CS intensity was set to $70 \%$ of $\mathrm{RMT}_{0.2 \mathrm{mV}}$. Stimulus adjustments were based on changes in the average $\mathrm{RMT}_{0.2 \mathrm{mv}}$ estimate obtained from three control channels after each pseudorandomization cycle. The test stimulus intensity was initially set to $120 \%$ of $\mathrm{RMT}_{0.2 \mathrm{mv}}$. It was then adjusted continuously using a step size of $1 \%$ MSO (doubled if two in a row stimuli "missed" the target), to maintain a target MEP of $0.2 \mathrm{mV}$ (Fisher et al., 2002). This was done independently for each SICI ISI condition, resulting in nine conditioned thresholds. To calculate the estimates of control and conditioned thresholds, the recorded log MEP response was plotted against the corresponding test stimulus intensity (Fisher et al., 2002). The 
test stimulus corresponding to the target MEP of $0.2 \mathrm{mV}$ was then estimated by regression analysis (Fisher et al., 2002). The regression analysis was weighted to account for the position of the data points, so that data points lying closer to the estimated regression line contributed more. Data points lying outside the linear part [0.02-2 mV] of the semi-logarithmic regression were excluded (Fisher et al., 2002). T-SICls were calculated as T-SICI = (conditioned threshold $-\mathrm{RMT}_{0.2 \mathrm{mv}}$ ) $/ \mathrm{RMT}_{0.2 \mathrm{mv}} \times 100 \%$ (Fisher et al., 2002). Negative values reflect cortical facilitation, and positive values reflect cortical inhibition. Variables of interest were $\mathrm{RMT}_{0.2 \mathrm{mV}}, \mathrm{T}-\mathrm{SICl}_{1 \mathrm{~ms}}, \mathrm{~T}-\mathrm{SICl}_{2.5 \mathrm{~ms}}, \mathrm{~T}-\mathrm{SICl}_{3 \mathrm{~ms}}$, $\mathrm{T}-\mathrm{SICl}$ averaged at ISI from 1 to $3.5 \mathrm{~ms}\left(\mathrm{~T}_{-}-\mathrm{SICl}_{1-3.5 \mathrm{~ms}}\right)$ and $\mathrm{T}-\mathrm{SICl}$ averaged at ISI from 1 to $7 \mathrm{~ms}\left(\mathrm{~T}-\mathrm{SICl}_{1-7 \mathrm{~ms}}\right)$. $\mathrm{RMT}_{0.2 \mathrm{mv}}$ was chosen as it reflects cortical excitability threshold. T-SICl $1-3.5 \mathrm{~ms}$ and $\mathrm{T}-\mathrm{SICl}_{1-7 \mathrm{~ms}}$ were investigated as predictors of general cortical inhibition.

\section{A-SICI protocol}

The A-SICl protocol was also managed by QTRACW and was initiated after $\mathrm{RMT}_{0.2 \mathrm{mv}}$ and $\mathrm{TS}_{1 \mathrm{mv}}$ had been estimated at the beginning of the examination (Fig. $3 A-C$ ). The same ISI conditions as in the T-SICI protocol were used. The same procedure for pseudorandomization of the nine $\mathrm{SICl}$ and three control conditions as in the T-SICI protocol was applied (Fig. $3 A$ ). The A-SICl protocol also stopped after each of the nine ISI conditions had been delivered ten times, and control stimuli at $\mathrm{TS}_{1 \mathrm{mv}} 30$ times.

As opposed to the continuous $\mathrm{RMT}_{0.2 \mathrm{mv}}$ estimation and continuous adjustment of the paired stimulus intensities at each ISI condition in T-SICl, in A-SICl the paired stimulus intensities for each condition remained fixed throughout the examination (Fig. $3 \mathrm{C}$ ). The CS intensity was fixed at $70 \%$ of the $\mathrm{RMT}_{0.2 \mathrm{mV}}$, and the test stimulus intensity was fixed at $\mathrm{TS}_{1 \mathrm{mv}}$.

Geometric means of MEPs were calculated for each paired stimulus condition (conditioned MEP) and each control stimulus (control MEP). The A-SICls were calculated as $\mathrm{A}-\mathrm{SICl}=($ conditioned $\mathrm{MEP} /$ control MEP $) \times 100 \%$ (Kujirai et al., 1993). Values above $100 \%$ reflect cortical facilitation, and values below $100 \%$ reflect cortical inhibition. The same variables of interest $\left(\mathrm{RMT}_{0.2 \mathrm{mv}}, \mathrm{A}-\mathrm{SICl}_{1 \mathrm{~ms}}\right.$, $\mathrm{A}-\mathrm{SICl}_{2.5 \mathrm{~ms}}, \mathrm{~A}-\mathrm{SICl}_{3 \mathrm{~ms}}, \mathrm{~A}-\mathrm{SICl}_{1-3.5 \mathrm{~ms}}$, and $\left.\mathrm{A}-\mathrm{SICl}_{1-7 \mathrm{~ms}}\right)$ were chosen as well as $\mathrm{TS}_{1 \mathrm{mV}}$.

\section{Exclusion of spontaneous muscle contraction}

For both protocols, the online gating threshold during recording was set to $15 \mu \mathrm{V}$ to exclude traces with contamination from spontaneous muscle contraction.

To exclude responses when the target muscle was not completely relaxed, online gating of prestimulus activation was used in both protocols. Sweeps in which negative EMG peaks exceeding $0.015 \mathrm{mV}$ were detected $270 \mathrm{~ms}$ before the magnetic stimuli were automatically discarded from the analysis.

\section{Statistical analysis}

Microsoft Excel version 16.16.15. was used for calculation of repeated measures ANOVA (rmANOVA) and
Student's paired $t$ test. Other statistical analyses were performed using the statistical software program $\mathrm{R}$ version 3.6.2 (2019-12-12) and OriginPro 2017 (OriginLab).

Normality was checked by quantile-quantile plots (QQplots) and histograms, and log-normally distributed data were log10-transformed.

Simple linear regression and calculation of correlation coefficients were applied for intraobserver method correlation analysis (Bland and Altman, 2003). $\mathrm{RMT}_{0.2 \mathrm{mV}}$ and SICl variables for each subject were calculated by averaging, either arithmetically (for normally distributed data) or geometrically (for log-normally distributed data), all measurements across all sessions. One sample $t$ test comparing to a control condition (0\% $\mathrm{RMT}_{0.2 \mathrm{mV}}$ for $\mathrm{T}-\mathrm{SICl}, 100 \%$ test MEP for A-SICl) was used to determine significant inhibition or facilitation at each ISI. The relationship between two $\mathrm{SICI}$ methods across all ISIs was described by fitting a linear curve $[y=a+b \times \log (10)$, where $a=$ intercept, $b=$ slope, $\mathrm{x}=$ mean group $\mathrm{A}-\mathrm{SICl}$, non-transformed].

Intraobserver repeatability was assessed by coefficient of repeatability (CR; Bartlett and Frost, 2008), intraobserver reliability was assessed by intraclass correlation coefficient (ICC; Koo and Li, 2016), and reproducibility of intrasession and intersession measurements were assessed by rmANOVA (Bland and Altman, 1999; Bland, 2015). An overview of the statistical terms is summarized in Table 1. Bland-Altman plots were constructed to examine for systematic observer bias (Bland and Altman, 1999; Bland, 2015). CR was defined as $C R=1.96 * \sqrt{2 \sigma_{w}}$ (Bland and Altman, 1986, 1999), and 95\% confidence interval (Cl) were calculated (Barnhart and Barboriak, 2009).

For normally distributed data, CR and Bland-Altman plots can be interpreted as the absolute difference between any two future replicate measurements estimated to be no greater than CR on $95 \%$ of occasions. However, this is not the case for CRs and Bland-Altman plots, which have been calculated with log-transformed data. Log-transformed CRs and Bland-Altman plots become dimensionless ratios on backtransformation (Bland and Altman, 2003), and can be interpreted as a relative difference between any two future replicate measurements estimated to be no greater than CR on $95 \%$ of occasions. A two-way random effects model with single-ratings, absolute-agreement [ICC $(2,1)]$ was applied to quantify ICC (Fleiss, 1999; de Vet et al., 2006; Streiner and Norman, 2008; Koo and Li, 2016; Brown et al., 2017). Reliability was defined as poor (ICC $<0.50$ ), moderate (ICC $0.50-0.749$ ), good (ICC $0.75-0.90$ ) or excellent (ICC > 0.90; Koo and Li, 2016).rmANOVA was also used to estimate between-subject and within-subject variance. If assumption of sphericity for rmANOVA was violated (Mauchly's sphericity test, $p<0.05$ ), Greenhouse-Geisser correction was applied. If significant effects were identified $(p<0.05)$, pairwise post hoc analysis with Bonferroni correction for multiple comparisons was applied.

Interobserver statistical parameters were calculated by applying the first examination by observer 1 in the corresponding session. If significant effects were identified $(p<0.05)$, pairwise post hoc analysis with Bonferroni correction for multiple comparisons was applied. Interobserver reliability was estimated by ICC. Student's $t$ test for paired 
Table 1: Overview of definitions, applicability and calculations of repeatability, reliability, and reproducibility

\begin{tabular}{|c|c|}
\hline Parameter & Definition \\
\hline Repeatability & $\begin{array}{l}\text { Variation in repeat measurements } \\
\text { made on the same subject under } \\
\text { identical conditions: Same method, } \\
\text { same observer, measurements are } \\
\text { taken in quick succession (Bartlett } \\
\text { and Frost, 2008). } \\
\text { Variation is ascribed to errors in the } \\
\text { measurement process (Bartlett and } \\
\text { Frost, 2008; Bland and Altman, } \\
\text { 1999). }\end{array}$ \\
\hline
\end{tabular}

Reliability Ratio of the subject variation compared with the total variation: subject variation and measurement error (variation in the measurement process; Bartlett and Frost, 2008).

A reliability of 1 indicates no measurement error and 0 indicates that all variation stems from measurement error (Koo and Li, 2016).

Reproducibility Variation in measurements made on the same subject under changing conditions:

Different methods or instruments, different observers, measurements being made at different timepoints, within which the "true" underlying variable could undergo non-negligible changes (Bartlett and Frost, 2008).

When to use
The CR can be used to study measurement preci-
sion (Bartlett and Frost, 2008). It is used when de-
cisions are made on an individual basis.
CR indicates how much two or more measurements
made on the same subject will vary on 95\% of oc-
casions (Bartlett and Frost, 2008). Thus, the higher
the measurement error, the higher the CR.

How to calculate $\mathrm{CR}=1.96 * \sqrt{2 \sigma_{w}}$, where $\sigma_{w}$ is withinsubject variance (Bartlett and Frost, 2008).

The ICC can be used to study the amount of measurement error in measurements made on the same subjects by different observers (interobserver reliability) or by a single observer (intraobserver reliability; Bartlett and Frost, 2008).

ICC measures how well subjects maintain their position within the group with repeated measurements (Streiner and Norman, 2008). This is important for sample size and power calculations in interventional studies (Fleiss, 1999; Brown et al., 2017) and provides some indication on a discriminative value of a test (de Vet et al., 2006).

As reliability ICC is a dimensionless ratio, ICC can be used to compare methods, whose measurements are on different scales (Koo and Li, 2016).

Reproducibility can be studied when measurements are made by different observers, with different methods or instruments, or at different timepoints (Bartlett and Frost, 2008).

Different statistical analysis methods have different assumptions. Choice of statistical analysis depends on study design, measurement scale, etc.
(SD of subjects' true values $)^{2} /[(\mathrm{SD}$ of subjects' true values $)^{2}+($ SD measurement error $\left.)^{2}\right]$ (Bartlett and Frost, 2008).

rmANOVA was used to study difference in timepoints.

Paired $t$ test (incl. correction for multiple comparison) was used to study interobserver differences. data were applied for comparison of interobserver measurements. 95\% Cl for interobserver estimates are not given, because two observers are too few to give useful estimates.

\section{Results}

\section{Data characteristics}

The cTMS and TT-TMS RMT $0.2 \mathrm{mv}$ were in general below $60 \% \mathrm{MSO}$, except for subjects S8 and $\mathrm{S} 11 . \mathrm{TS}_{1 \mathrm{mV}}$ was on average $119.3 \%$ (range 109.9-144.7\%) of $\mathrm{RMT}_{0.2 \mathrm{mv}}$ for cTMS. Most of the subjects exhibited cortical inhibition at $\mathrm{SICl}_{1 \mathrm{~ms}}, \mathrm{SICl}_{2.5 \mathrm{~ms}}$ and $\mathrm{SICl}_{3 \mathrm{~ms}}$ with cTMS and TT-TMS (Figs. $4,5)$. Subject S14 (male, age: 75 years) exhibited cortical facilitation with both TMS methods throughout all sessions at $\mathrm{SICl}_{1 \mathrm{~ms}}, \mathrm{SICl}_{2.5 \mathrm{~ms}}$, and $\mathrm{SICl}_{3 \mathrm{~ms}}$. Average skin temperature for both methods was $35.4^{\circ} \mathrm{C}$ [range $34.5-36.4^{\circ} \mathrm{C}$ ]. A-SICls were log-normally distributed. All other TMS parameters were normally distributed. Description of data are given in Table 2 .

\section{Method correlation (intraobserver measurements)}

On a group level, significant inhibition was seen at ISIs 1-3 ms with TT-TMS and at ISIs 1-4 ms with cTMS, with two distinct peaks observed at 1 and $2.5 \mathrm{~ms}$ with both techniques (Fig. 6). Meanwhile, there was significant facilitation at ISI $7 \mathrm{~ms}$ with both methods.

At individual or averaged ISIs, both intraobserver $\mathrm{RMT}_{0.2 \mathrm{mV}}$ and $\mathrm{SICl}$ measurements obtained with cTMS and TT-TMS all correlated significantly (Fig. 7). The correlation coefficient, $r$, for SICls ranged from $0.79(95 \% \mathrm{Cl}$ [0.52-0.92]) to $0.82(95 \% \mathrm{Cl}[0.56-0.93])$ and was 0.98

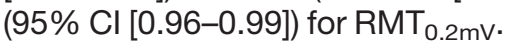

On a group level, a strong linear relationship between $\mathrm{T}$ $\mathrm{SICl}$ and log-transformed A-SICl was observed across the whole range of tested ISIs, and this relationship was maintained throughout the experimental days as well as the time of the day (Fig. 8). However, there was considerable variability in this relationship in individual subjects with some discordance (i.e., one showing inhibition, another, facilitation) between the techniques at some ISIs (Fig. 9).

\section{Repeatability (intraobserver measurements)}

Back-transformed $\mathrm{CR}$ for $\mathrm{A}-\mathrm{SICl}_{1 \mathrm{~ms}}, \mathrm{~A}-\mathrm{SICl}_{2.5 \mathrm{~ms}}$, and $\mathrm{A}-\mathrm{SICl}_{3 \mathrm{~ms}}$ ranged from $2.6(95 \% \mathrm{Cl}[2.1-4.6])$ to $7.2(95 \%$ 

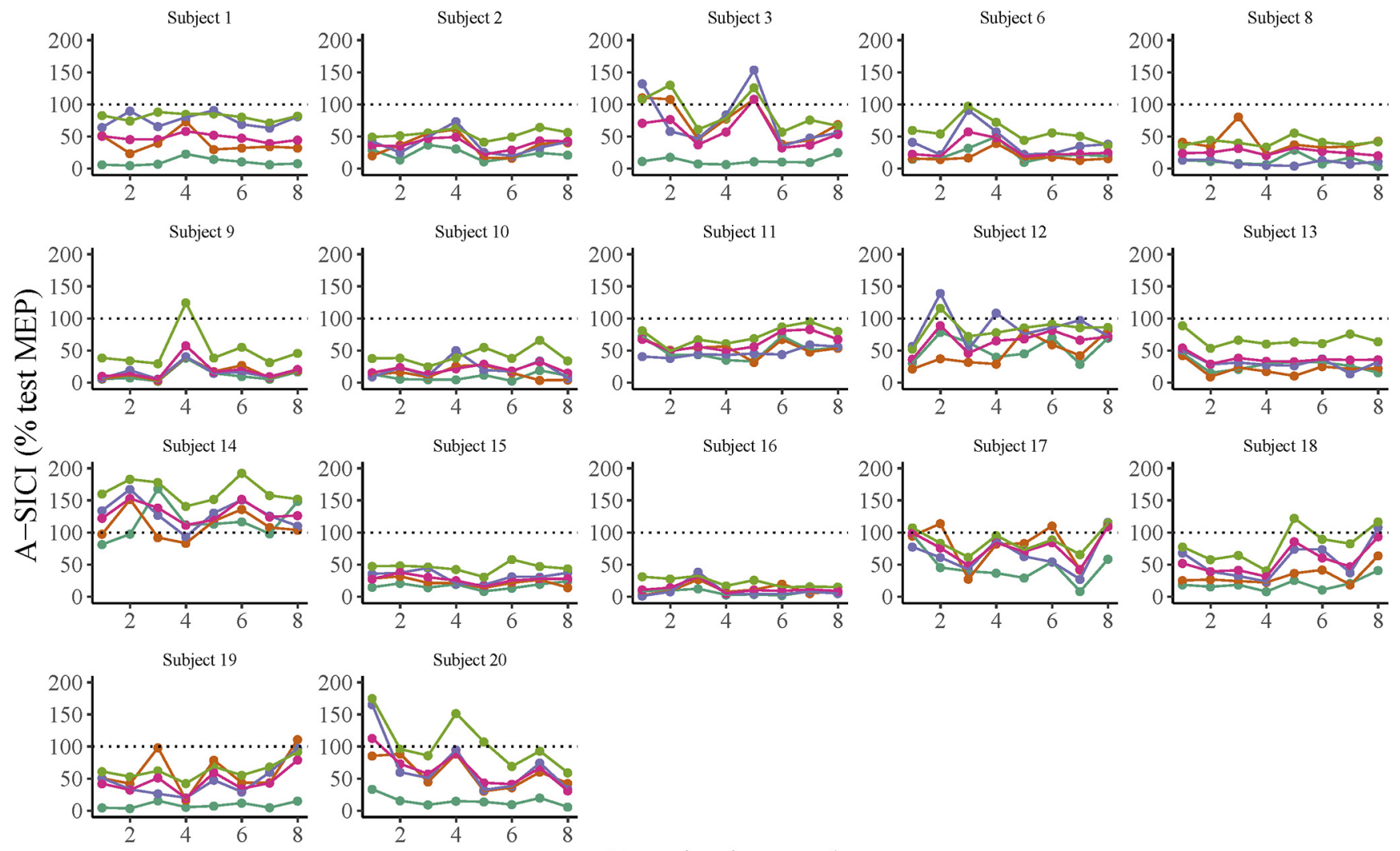

Examination number

ISI (in $\mathrm{ms}) \rightarrow 1 \mathrm{~ms} \rightarrow 2.5 \mathrm{~ms} \rightarrow 3 \mathrm{~ms} \rightarrow 1-3.5 \mathrm{~ms} \rightarrow 1-7 \mathrm{~ms}$

Figure 4. Intraobserver A-SICl examinations for all subjects. $x$-axis: examination number. $y$-axis: $\mathrm{SICI}$ values measured with cTMS. Horizontal dotted line denotes threshold for cortical facilitation (A-SICl $>100$, in \% MEP) or inhibition $(\mathrm{A}-\mathrm{SICl}<100$, in \% test $\mathrm{MEP})$. ISI of 1, 2.5, 3, 1-3.5, and 1-7 ms are depicted in different colors. Each subfigure denotes measurements from one individual subject. Subject number 4 (missing data point), subject number 5 (undetectable MEP), and subject number 7 (unrelaxed hand muscles) were excluded.

Cl [4.5-21.8]) on both days (Table 3). Thus, the difference between any two future examinations is estimated to be no greater than 2.6- to 7.2-fold difference on $95 \%$ of occasions for $\mathrm{A}-\mathrm{SICl}_{1 \mathrm{~ms}}, \mathrm{~A}-\mathrm{SICl}_{2.5 \mathrm{~ms}}$, and $\mathrm{A}-\mathrm{SICl}_{3 \mathrm{~ms}}$. CR for $\mathrm{T}-\mathrm{SICl}_{1 \mathrm{~ms}}, \mathrm{~T}-\mathrm{SICl}_{2.5 \mathrm{~ms}}$, and $\mathrm{T}-\mathrm{SICl}_{3 \mathrm{~ms}}$ ranged from $9.4 \%$ to $17.3 \% \mathrm{RMT}_{0.2 \mathrm{mv}}$ on both days (Table 3 ), meaning that the absolute difference between any two future examinations is estimated to be no greater than $9.4-17.3 \%$ $\mathrm{RMT}_{0.2 \mathrm{mv}}$ on $95 \%$ of occasions for $\mathrm{T}-\mathrm{SICl}_{1 \mathrm{~ms}}, \mathrm{~T}-\mathrm{SICl}_{2.5 \mathrm{~ms}}$ and $\mathrm{T}-\mathrm{SICl}_{3 \mathrm{~ms}}$.

CRs tended to be lower for averaged SICls $\left(\mathrm{SICl}_{1-3.5 \mathrm{~ms}}\right.$ and $\left.\mathrm{SICl}_{1-7 \mathrm{~ms}}\right)$ compared with the non-averaged SICls $\left(\mathrm{SICl}_{1 \mathrm{~ms}}, \mathrm{SICl}_{2.5 \mathrm{~ms}}\right.$, and $\left.\mathrm{SICl}_{3 \mathrm{~ms}}\right)$. Also, CRs tended to be lower for morning $\mathrm{SICl}$ measurements compared with afternoon $\mathrm{SICl}$ measurements on both days. CRs for $\mathrm{TS}_{1 \mathrm{mV}}$ tended to be higher than for $\mathrm{RMT}_{0.2 \mathrm{mV}}$ in cTMS (Table 3).

\section{Intraday and interday reliability (intraobserver measurements)}

On day $1, \mathrm{~A}-\mathrm{SICl}$ morning reliability ranged from moderate to good, afternoon reliability ranged from poor-to- moderate, and intraday reliability was moderate-to-good (Fig. 10A). On day 2, A-SICl morning reliability was good, and afternoon and intraday reliability both ranged from moderate to good (Fig. 10B).

On day 1, T-SICl morning reliability was good, and afternoon and intraday reliability ranged from moderate to good (Fig. 10C). T-SICl reliability ranged from moderate to good on day 2 (Fig. 10D).

Interday reliability of all SICI measurements was moderate-to-good (Fig. 11). Intraday and interday reliability of all $\mathrm{RMT}_{0.2 \mathrm{mv}}$ and $\mathrm{TS}_{1 \mathrm{mv}}$ ranged from good to excellent (Figs. 10, 11).

\section{Reproducibility (intraobserver measurements)}

None of the Bland-Altman plots for A-SICl measurements revealed ratios significantly different from 0 , except for the $\mathrm{A}-\mathrm{SICl}_{2.5 \mathrm{~ms}}$ ratio $\frac{A-S / C l_{2.5 \mathrm{~ms}} \text { examination } 7}{A-S / C l_{2.5 m s} \text { examination } 8}=0.73$ $(95 \% \mathrm{Cl}[0.56-0.94])$ in the afternoon session on day 2. rmANOVA revealed that between-subject variation accounted for the largest part of total intersession variation, and that between-subject differences were significant for 

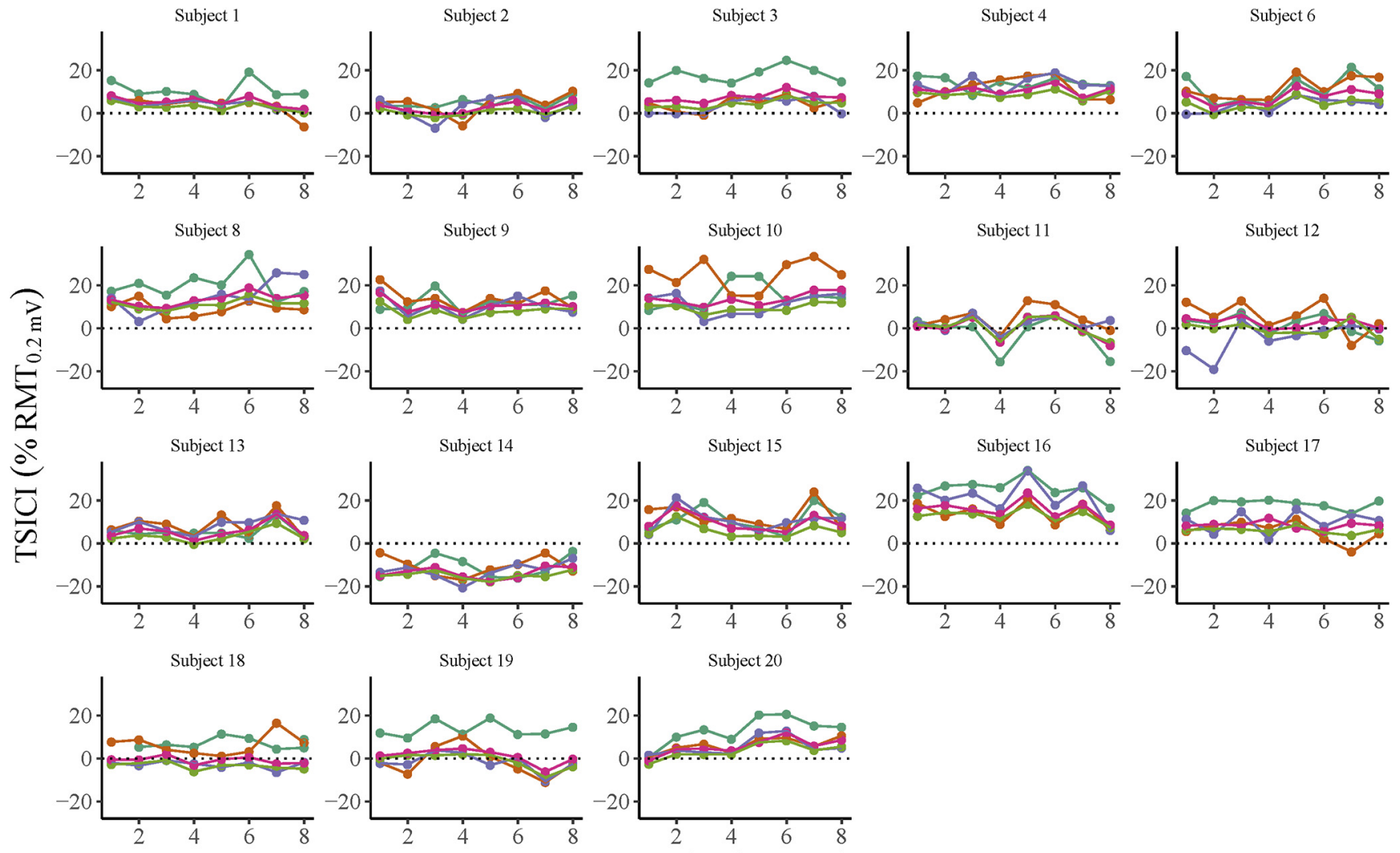

Examination number

ISI (in $\mathrm{ms}) \rightarrow 1 \mathrm{~ms} \rightarrow 2.5 \mathrm{~ms} \rightarrow 3 \mathrm{~ms} \rightarrow 1-3.5 \mathrm{~ms} \rightarrow 1-7 \mathrm{~ms}$

Figure 5. Intraobserver T-SICI examinations for all subjects. $x$-axis: examination number. $y$-axis: $\mathrm{SICI}$ measured with $\Pi \mathrm{T}$-TMS. Horizontal dotted line denotes threshold for cortical facilitation ( $\mathrm{T}-\mathrm{SICI}<0$, in \% $\mathrm{RMT}_{0.2 \mathrm{mv}}$ ) or inhibition $(\mathrm{T}-\mathrm{SICl}>0$, in \% $\mathrm{RMT} 0.2 \mathrm{mv}$ ). ISI of 1, 2.5, 3, 1-3.5, and 1-7 ms are depicted in different colors. Each subfigure denotes measurements from one individual subject. Subject number 5 (undetectable MEP) and subject number 7 (unrelaxed hand muscles) were excluded.

Table 2: Description of intraobserver measurements

\begin{tabular}{|c|c|c|c|c|c|c|c|}
\hline Method & Parameter & Sample size & Total number of measurements & Median $[\mathrm{IQR}]^{\mathrm{a}}$ & Mean & $\pm \mathrm{SE}^{\mathrm{b}}$ & SD \\
\hline \multirow[t]{5}{*}{$\overline{\text { cTMS }}$} & $\mathrm{A}-\mathrm{SICl}_{1 \mathrm{~ms}}$ & 17 & 136 & $16.1[16.4]$ & & & \\
\hline & $\mathrm{A}-\mathrm{SICl}_{3 \mathrm{~ms}}$ & 17 & 136 & $40.7[31.5]$ & & & \\
\hline & $\mathrm{A}-\mathrm{SICl}_{1-7 \mathrm{~ms}}$ & 17 & 1224 & 50.9 [32.2] & & & \\
\hline & $\mathrm{RMT}_{0.2 \mathrm{mv}}$ & 17 & 136 & & 54.2 & $( \pm 1.94)$ & 8.0 \\
\hline & $\mathrm{TS}_{1 \mathrm{mV}}$ & 17 & 136 & & 64.7 & $( \pm 2.88)$ & 11.9 \\
\hline \multirow{4}{*}{ TT-TMS } & $\mathrm{T}-\mathrm{SICl}_{3 \mathrm{~ms}}$ & 18 & 144 & & 5.5 & $( \pm 1.89)$ & 8.0 \\
\hline & $\mathrm{T}_{-} \mathrm{SICl}_{1-3.5 \mathrm{~ms}}$ & 18 & 864 & & 5.9 & $( \pm 1.60)$ & 6.8 \\
\hline & $\mathrm{T}-\mathrm{SICl}_{1-7 \mathrm{~ms}}$ & 18 & 1296 & & 3.5 & $( \pm 1.51)$ & 6.4 \\
\hline & $\mathrm{RMT}_{0.2 \mathrm{mV}}$ & 18 & 144 & & 55.2 & $( \pm 1.98)$ & 8.4 \\
\hline
\end{tabular}

Measurements of TMS parameters by observer 1 . Sample size $n=18$ for TT-TMS and $n=17$ for cTMS. Each subject was examined eight times by each method. One subject (S4) was examined seven times with cTMS and eight times with TT-TMS. SICl was measured with the A-SICl and the T-SICI parallel protocol. Only values measured at ISIs $1 \mathrm{~ms}$ (SICl1ms), $2.5 \mathrm{~ms}$ (SICl2.5ms), $3 \mathrm{~ms}$ (SICI3ms), 1-3.5 ms (SICl1-3.5ms), and 1-7 ms (SICl1-7ms) are depicted. Each TMS parameter was measured twice in each session.

${ }^{a}$ Point estimates for each subject were calculated as geometric means of their measurements. Data are displayed as medians with interquartile ranges [IQR] of subjects' point estimates.

${ }^{b}$ Normally distributed data for each subject was arithmetically averaged to calculate group mean ( \pm SE) and SD. 


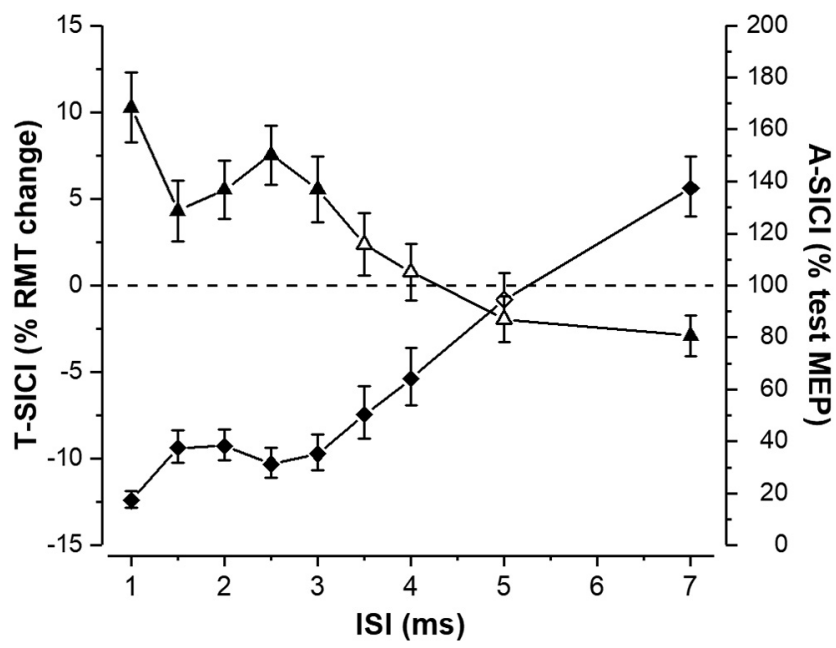

Figure 6. Mean group TT-TMS and cTMS SICl curves. Group means of $\mathrm{T}-\mathrm{SICl}$ (arithmetic, depicted by triangles) and A-SICI (geometric, depicted by diamonds) were calculated by averaging all 18 individual subjects' means across all measurements. Error bars represent SEM ( \pm SEM for $\mathrm{T}-\mathrm{SICI}, \times / \div$ SEM for A-SICI). Filled symbols represent a significant inhibition or facilitation when compared with the control condition $\left(0 \% \mathrm{RMT}_{0.2 \mathrm{mV}}\right.$ for TT-TMS and $100 \%$ test MEP for cTMS; one-sample $t$ test $p<0.05$ ).

all TMS parameters. None of the TMS examinations differed significantly from another examination, except for $\mathrm{RMT}_{0.2 \mathrm{mV}}$ in cTMS $\left(F_{(4.13,66)}=2.53, p=0.046\right), \mathrm{TS}_{1 \mathrm{mV}}$ $\left(F_{(16,7)}=2.2, p=0.038\right)$, and $\mathrm{T}-\mathrm{SICl}_{3 \mathrm{~ms}}\left(F_{(17,7)}=2.49, p=\right.$ $0.02)$. After correcting for multiple comparisons using Bonferroni correction, examination 4 differed significantly from examination 6 for $\mathrm{T}-\mathrm{SICl}_{3 \mathrm{~ms}}(p=0.00035)$, and examination 1 differed significantly from examination 4 for cTMS $\mathrm{TS}_{1 \mathrm{mv}}(p=0.00174)$, but none of the cTMS RMT $\mathrm{R}_{0.2 \mathrm{mv}}$ examinations differed significantly from another.

\section{Interobserver reliability and reproducibility}

Among interobserver reproducibility measurements, a significant difference was observed in the $\mathrm{A}-\mathrm{SICl}_{2.5 \mathrm{~ms}}$ ratio $\frac{\mathrm{A}-\mathrm{SICl}_{2.5 \mathrm{~ms}} \text { observer } 2}{\mathrm{~A}-\mathrm{SICl}_{2.5 \mathrm{~ms}} \text { observer } 1}=1.56(95 \% \mathrm{Cl}[1.28-2.17])$, $p=0.0103$ (Table 4). None of the other comparisons (neither T-SICl differences, nor A-SICI ratios) were significantly different between observer 1 and observer 2 .

Interobserver reliability for SICls ranged from poor-to-moderate, and good to excellent for RMT $\mathrm{R}_{0.2 \mathrm{mv}}$ and $\mathrm{TS}_{1 \mathrm{mV}}$ (Fig. 12).

\section{Discussion}

The present study elaborates multiple aspects of testretest reliability of two emerging TMS protocols, including intraobserver and interobserver, intraday and interday reliability as well as diurnal influences on it. The present work extends current knowledge of the utility of these two novel SICI protocols.

\section{Comparability between the techniques}

Earlier studies have compared parallel TT-TMS with cTMS for SICI with either multiple CS intensities and single ISI (Samusyte et al., 2018) or a single CS intensity and multiple ISIs (Tankisi et al., 2021a).

Despite some technical differences in the parallel threshold-tracking paradigm (such as tracking mode or maximum tracking step size), a good correlation between $\mathrm{A}-\mathrm{SICl}$ and $\mathrm{T}-\mathrm{SICl}$ measurements was observed in these studies both within and across tested $\mathrm{SICl}$ conditions, suggesting that both techniques reflect largely similar underlying physiological mechanisms, at least in healthy volunteers.

In the present study, a good correlation between $\mathrm{SICl}$ measurements obtained with the two protocols both at individual ISIs and averaged SICI was observed across subjects. Furthermore, a linear relationship between T-SICI and log-transformed A-SICl was found across the whole range of tested ISIs. On a group level, this relationship appeared to remain stable throughout the day or different experimental days. Nevertheless, considerable interindividual variability in $\mathrm{A}-\mathrm{SICl} / \mathrm{T}-\mathrm{SICl}$ slopes was observed, which is similar to earlier findings comparing SICls at multiple conditioning intensities (Samusyte et al., 2018). In several subjects (Fig. 9), a discrepancy between the methods was seen at some ISIs, which is probably reflected in the slightly different duration of inhibition in the group (1$3 \mathrm{~ms}$ for $\mathrm{T}-\mathrm{SICl}$ vs $1-4 \mathrm{~ms}$ for A-SICl; Fig. 6). This cannot be explained by differences in CS intensity as it was set to $70 \%$ of tracked $\mathrm{RMT}_{0.2 \mathrm{mV}}$ for both techniques. Although it is common to adjust CS based on active motor threshold or conventional RMT of $0.05 \mathrm{mV}$ (Rossini et al., 2015), $\mathrm{RMT}_{0.2 \mathrm{mv}}$ was used in the present study to ensure activation of comparable inhibitory neuron populations with both methods as $\mathrm{SICl}$ is known to vary depending on conditioning intensity (Kujirai et al., 1993; Vucic et al., 2009). However, because of the intrinsic differences in the techniques (predetermined test MEP size dependent on a constant test stimulus in cTMS versus predetermined conditioned MEP size resulting in varying test stimuli across ISIs in TT-TMS), the upper motor neuron populations tested by the two techniques may differ.

\section{Repeatability}

CR can be used to estimate measurement error (Bartlett and Frost, 2008). In statistics, "measurement error" refers to the inherent continuous natural variation that occurs with repeated measurements of the same biological quantity in a subject. The measurement error may include natural biological variability in the subject and variability in the measurement method (Bland, 2015). Thus, measurement error does not refer to a mistake made during the examination, e.g., when an estimate is written down incorrectly (Bland, 2015).

One way to report measurement error is to estimate how much any two future measurements made on the same subject are expected to differ. This estimate may also be called "within-subject variation": second measurements on the same subject are not expected to differ systematically from the first measurement, as this would indicate the values were not true replicates (Bland and Altman, 1999). Hence, the possibility of bias between measurements is excluded, and the measurement error 
A

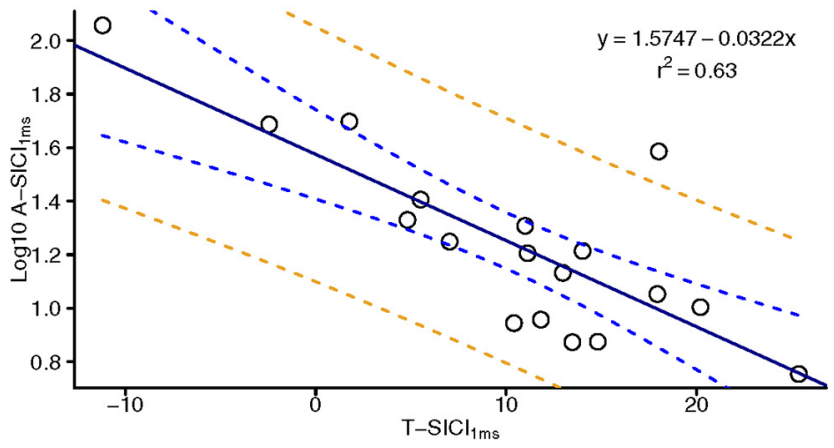

C

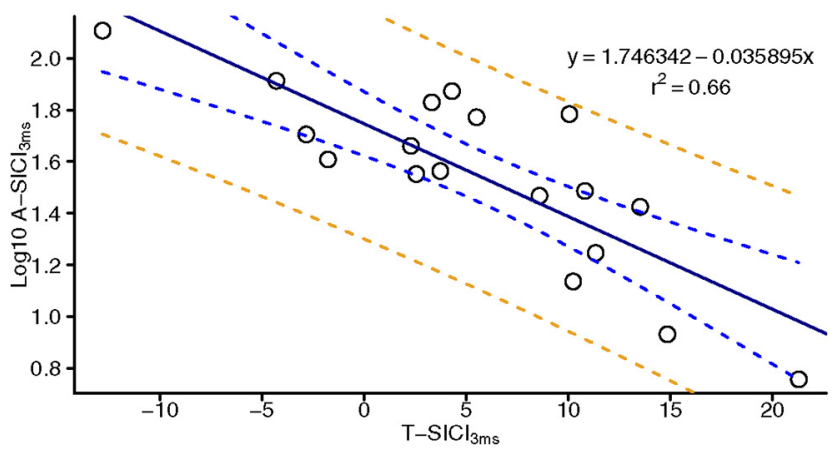

E

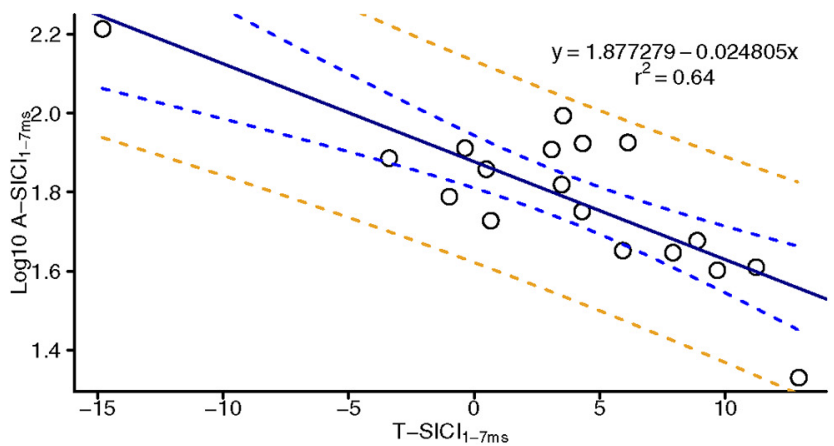

B

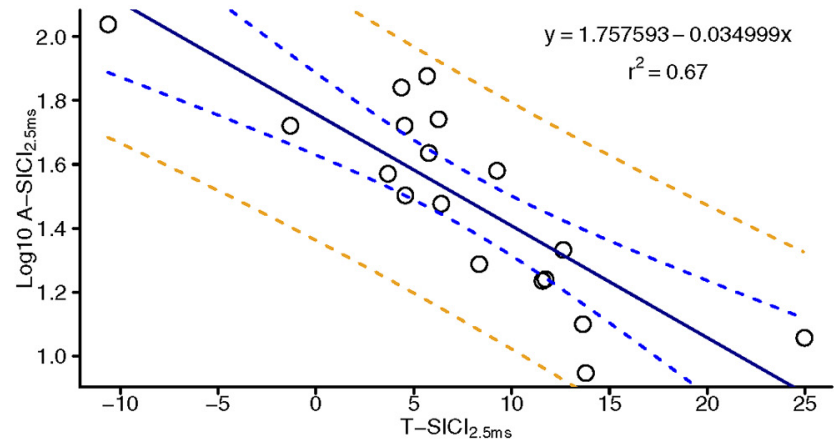

D

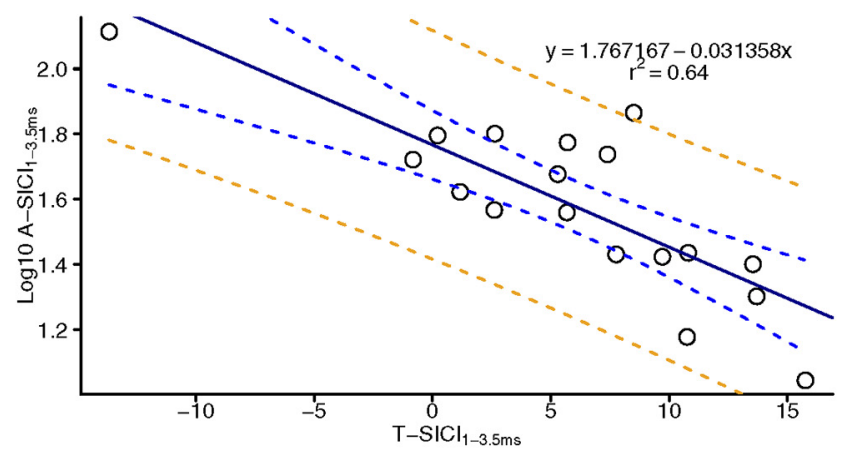

$\mathbf{F}$

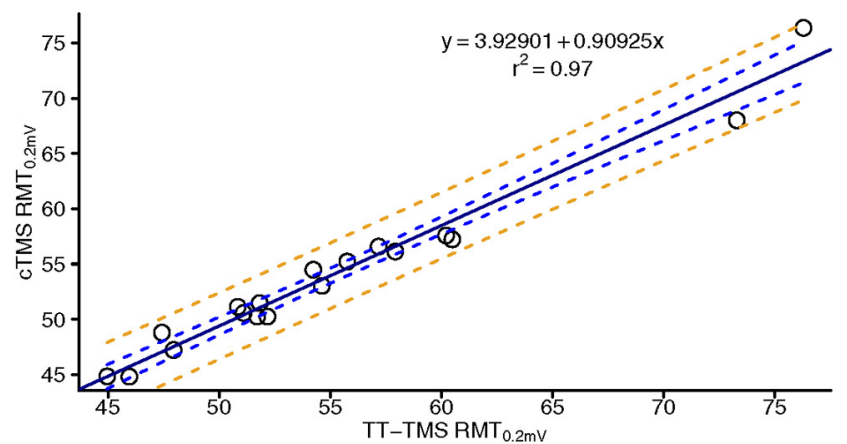

Figure 7. Regression of cTMS and TT-TMS. Simple regression of intraobserver (observer 1) cTMS and TT-TMS parameters. $x$-axis: $\mathrm{T}$-SICl measured with TT-TMS. $y$-axis: Log10 A-SICI measured with cTMS. Regressions depict SICI ISIs measured at 1 ms $(\boldsymbol{A})$, $2.5 \mathrm{~ms}(\boldsymbol{B}), 3 \mathrm{~ms}(\boldsymbol{C}), 1-3.5 \mathrm{~ms}(\boldsymbol{D})$, and 1-7 ms $(\boldsymbol{E})$. RMT $0.2 \mathrm{mv}$ measured with TT-TMS and cTMS $(\boldsymbol{F})$. Full line (black) denotes simple linear regression line, blue dashed line denotes $95 \% \mathrm{Cl}$ for regression line, and orange dashed line denotes $95 \%$ prediction intervals for the regression line. Measurements from all eight examinations for all subjects for each TMS parameter were averaged. The TMS regression $r^{2}$ are denoted in the upper right corner. All cTMS and TT-TMS measurements correlated significantly.

depends only on the within-subject variation. Withinsubject variation is therefore the same as the variation of the measurement error (Bartlett and Frost, 2008). $\mathrm{CR}$ estimates measurement error by quantifying the size of the differences between any two future measurements made on the same subject on $95 \%$ of occasions (Bland and Altman, 1999; Bartlett and Frost, 2008). Thus, the higher the $C R$, the higher the measurement error.

Measurement error in the present study may be ascribed to variation in coil placement and coil angling during the examination. The TMS coil was kept manually in place, and it was observed that even a slight change in coil angling by a few degrees or millimeters, either because of head movement by the subject or coil movement by the observer, could stimulate nearby muscles on the same hand, thereby possibly introducing measurement error. Likewise, the location of motor hotspot in the beginning of each measurement might have been subject to variation as well. The location was done manually, without a navigation system. It is possible that the exact same coil position and coil angling was not achieved in each consecutive examination, which might have contributed to the observed measurement error.

Measurement error in the present study may also be ascribed to biological variation in the subjects. Although 

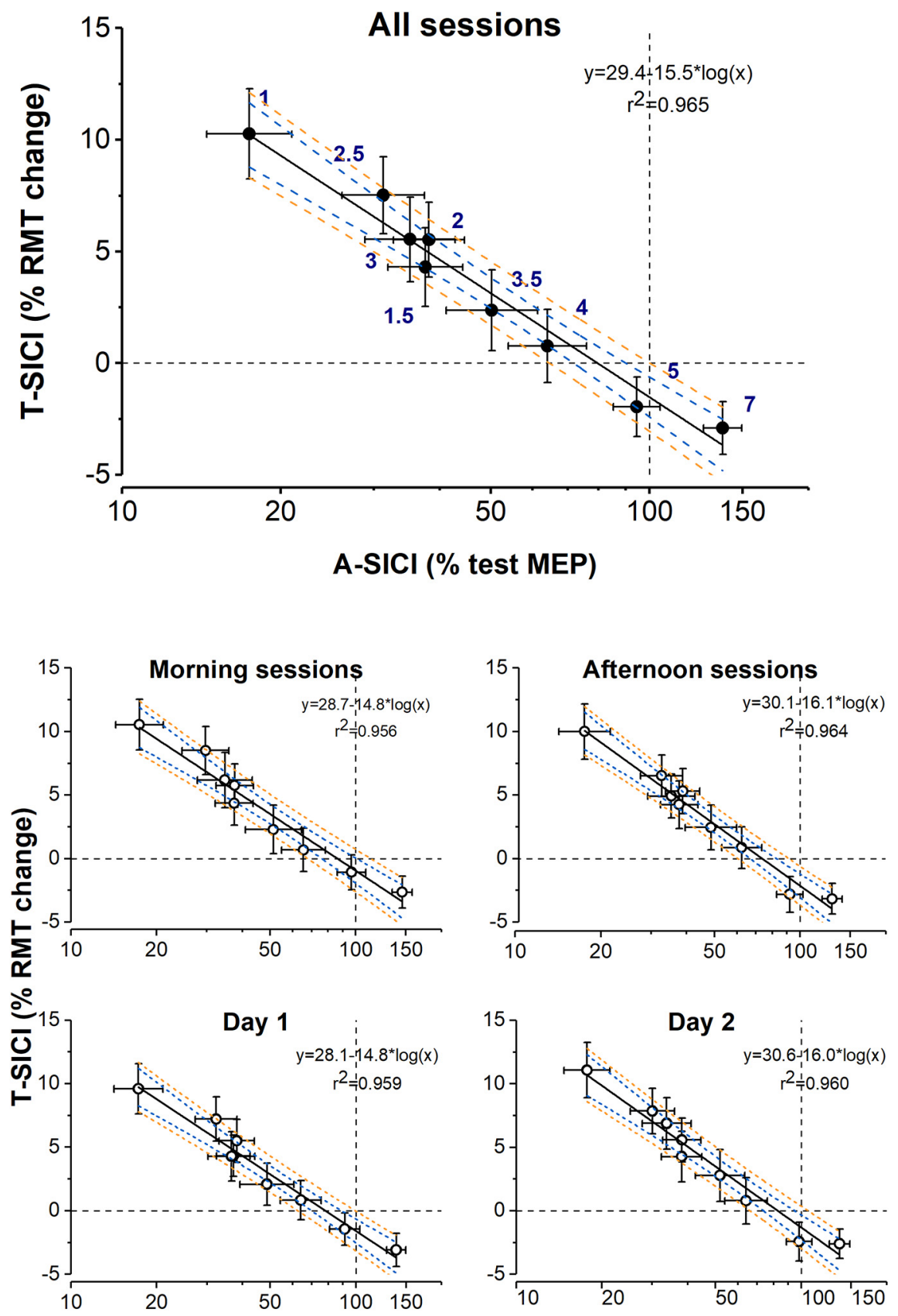

A-SICI (\% test MEP)

Figure 8. Relationship between mean group $\mathrm{A}-\mathrm{SICl}$ and $\mathrm{T}-\mathrm{SICl}$ curves. Group means of T-SICl (arithmetic) are plotted against A$\mathrm{SICl}$ (geometric) at matching ISIs. $x$-axis $\left(\log _{10}\right.$ scale): A-SICl obtained by cTMS. $y$-axis (linear scale): T-SICl obtained by TT-TMS. T$\mathrm{SICl}$ and $\mathrm{A}-\mathrm{SICl}$ group means were calculated by averaging all 18 individual subjects' means across all measurements (all sessions), across measurements taken on the same time of day (morning and afternoon sessions), or the same experimental day (day 1 and day 2). Error bars represent SEM ( \pm SEM for T-SICI, $\times / \div$ SEM for A-SICI). A linear relationship (denoted by black solid line, blue dashed line $95 \% \mathrm{Cl}$ for regression line, and orange dashed line $95 \%$ prediction intervals for regression line) was observed between $\mathrm{T}-\mathrm{SICl}$ and log-transformed A-SICl across ISIs (as indicated by navy numbers in the top panel), and was maintained throughout the experimental days as well as different time of the day.

the examinations were done in quick succession, the long duration of each session may have decreased subject alertness. A recent study demonstrated that spontaneously occurring fluctuations in alertness modulate cortical reactivity and MEP amplitude over relatively short durations in awake subjects (Noreika et al., 2020). If these findings can be extrapolated to the present study, then fluctuations in subject alertness may have contributed to biological variability, and thus measurement error in the present study. Furthermore, 

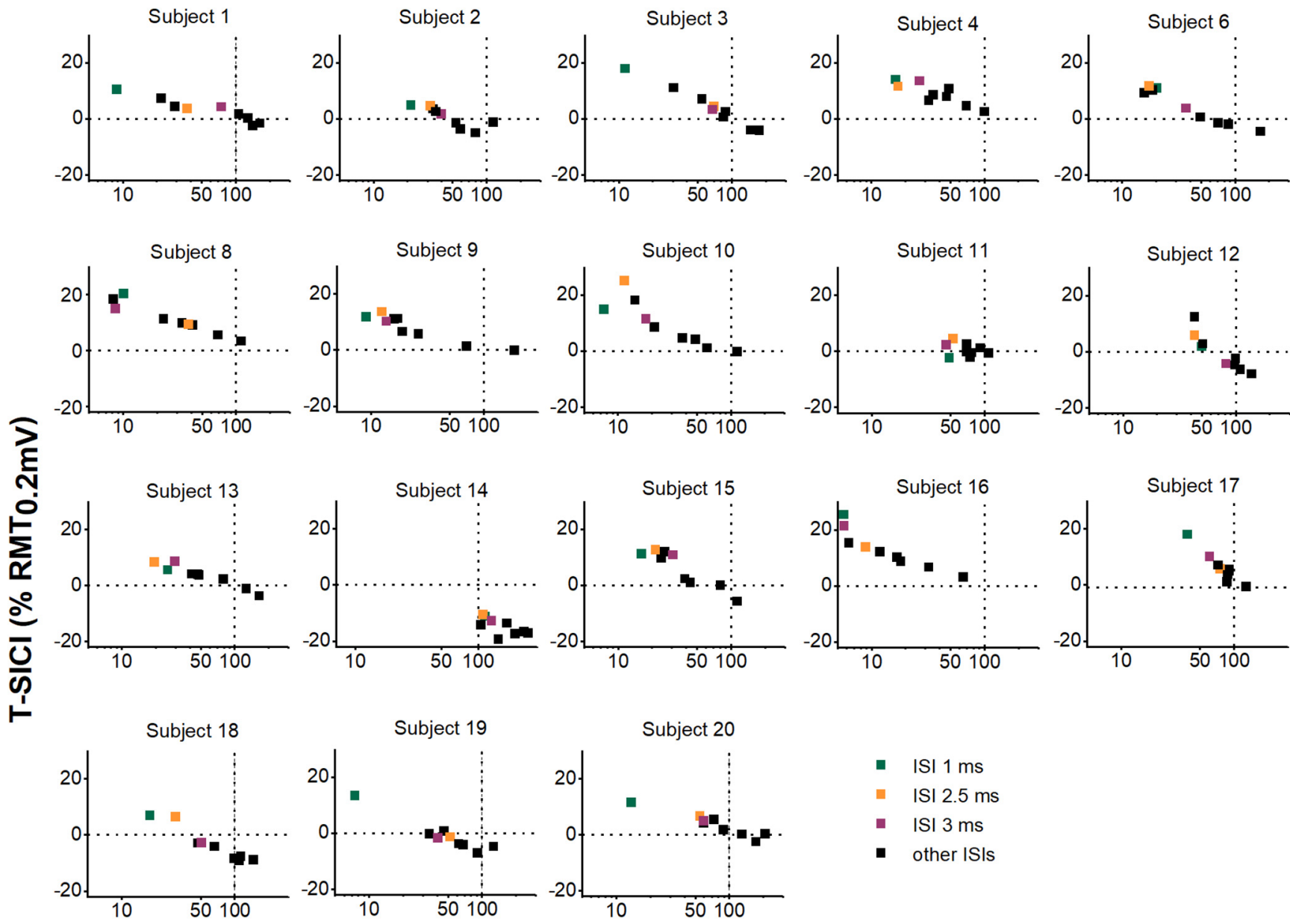

\section{A-SICl (\% test MEP)}

Figure 9. Relationship between A-SICI and T-SICI curves in individual subjects. Individual subject means of T-SICI (arithmetic) are plotted against A-SICI (geometric) at matching ISIs (calculated by averaging individual subjects' means across all measurements). Black dashed lines indicate control conditions (0\% RMT $0.2 \mathrm{mV}$ for T-SICI, $100 \%$ test MEP for A-SICl). $x$-axis (log 10 scale): A-SICl obtained by cTMS. $y$-axis (linear scale): T-SICI obtained by TT-TMS. In many subjects, the relationship between log-A-SICI and T-SICI appeared to be linear or near-linear. However, in some there seemed to be a "floor effect" with cTMS that was overcome by TTTMS (e.g., subject 12, subject 15); in others, no apparent correlation between the techniques was seen (e.g., subject 11, subject 14).

underlying oscillations in brain activity may affect TMS measurements (Zrenner et al., 2018; De Goede and Van Putten, 2019) and further contribute to the measurement error.

In the present study, the repeatability of $\mathrm{RMT}_{0.2 \mathrm{mV}}$ is in line with studies that used probabilistic methods to determine the conventional RMT with a $0.05 \mathrm{mV}$ cutoff (Beaulieu et al., 2017). Meanwhile, the intraday CRs of $\mathrm{RMT}_{0.2 \mathrm{mV}}$ and $\mathrm{TS}_{1 \mathrm{mV}}$ are comparable to the previous study, which employed threshold-tracking and reported CRs of $5.5 \%$ and $10 \%$ MSO, respectively (Samusyte et al., 2018). The repeatability of $\mathrm{A}-\mathrm{SICl}$ in the present study cannot be directly compared with previous studies which reported a wide range of CRs $(17-147 \%$ test MEP; Fleming et al., 2012; Ngomo et al., 2012; Schambra et al., 2015; Samusyte et al., 2018). Because of non-normality, log-transformed A-SICl was used to calculate CRs. The back-transformed CRs are dimensionless and therefore it is not straightforward to apply them in clinical practice (Schambra et al., 2015). However, conceptually CRs are similar to Bland-Altman's limits of agreement, which, if calculated with log-transformed values and then backtransformed, indicate a ratio to the value on the $x$-axis (Bland and Altman, 1999). Thus, measurement error of log-transformed values should be interpreted as a relative difference or fold-change between any two future measurements.

Meanwhile, the CRs for T-SICl were high when compared with their respective means. This shows that in an average subject with inhibition on the initial recording, some degree of facilitation could be observed with repeated testing, representing an expected variation (be it technical or biological). This is in keeping with previous reports of rather poor repeatability of $\mathrm{T}-\mathrm{SICI}$ measurements 
Table 3: Repeatability of cTMS and TT-TMS parameters

\begin{tabular}{|c|c|c|c|c|c|c|c|c|c|c|}
\hline \multirow[b]{3}{*}{ Method } & \multirow{3}{*}{$\begin{array}{l}\text { TMS } \\
\text { Parameter }\end{array}$} & \multirow{3}{*}{$\begin{array}{l}\text { Sample } \\
\text { size }(n)\end{array}$} & \multicolumn{8}{|c|}{ CR $[95 \% \mathrm{Cl}]$} \\
\hline & & & \multicolumn{4}{|c|}{ Day 1} & \multicolumn{4}{|c|}{ Day 2} \\
\hline & & & CR & $95 \% \mathrm{Cl}$ & CR & $95 \% \mathrm{Cl}$ & $\mathrm{CR}$ & $95 \% \mathrm{Cl}$ & $\mathrm{CR}$ & $95 \% \mathrm{Cl}$ \\
\hline \multirow{4}{*}{ cTMS } & $\mathrm{A}-\mathrm{SICl}_{2.5 \mathrm{~ms}}$ & 17 & 3.4 & [2.5-6.7] & 7.2 & [4.5-21.8] & 2.6 & [2.1-4.6] & 3.2 & {$[2.4-6.0]$} \\
\hline & $\mathrm{A}-\mathrm{SICl}_{3 \mathrm{~ms}}$ & 17 & 4.4 & [3.1-10.2] & 6.0 & [3.9-16.6] & 2.7 & [2.2-4.8] & 4.1 & [2.9-8.9] \\
\hline & $\mathrm{A}-\mathrm{SICl}_{1-3.5 \mathrm{~ms}}$ & 17 & 2.0 & {$[1.7-3.0]$} & 4.9 & [3.4-12.4] & 2.2 & [1.8-3.5] & 2.5 & [2.1-4.3] \\
\hline & $\mathrm{TS}_{1 \mathrm{mV}}$ & 17 & 8.6 & [6.6-13.5] & 13.3 & [10.1-20.7] & 10.1 & {$[7.8-15.8]$} & 12.8 & [9.8-20.0] \\
\hline \multirow[t]{5}{*}{ TT-TMS } & $\mathrm{T}-\mathrm{SICl}_{1 \mathrm{~ms}}$ & 18 & 10.2 & [7.8-15.7] & 15.4 & [11.9-23.8] & 14.5 & [11.2-22.3] & 13.8 & [10.7-21.3] \\
\hline & $\mathrm{T}-\mathrm{SICl}_{2 . \mathrm{ms}}$ & 18 & 9.4 & [7.2-14.5] & 13.5 & [10.4-20.8] & 12.9 & [9.9-19.9] & 17.3 & [13.4-26.7] \\
\hline & $\mathrm{T}-\mathrm{SICl}_{3 \mathrm{~ms}}$ & 18 & 13.5 & [10.4-20.8] & 13.5 & [10.4-20.8] & 9.9 & [7.7-15.3] & 12.2 & {$[9.4-18.8]$} \\
\hline & $\mathrm{T} \mathrm{SICl}_{1-3.5 \mathrm{~ms}}$ & 18 & 8.0 & [6.2-12.3] & 8.5 & [6.6-13.2] & 7.9 & [6.1-12.2] & 9.0 & [6.9-13.9] \\
\hline & $\mathrm{T}-S I C l_{1-7 \mathrm{~ms}}$ & 18 & 6.9 & [5.4-10.8] & 7.6 & [5.9-11.7] & 6.4 & [4.9-9.9] & 8.4 & [6.5-13.0] \\
\hline
\end{tabular}

For intraobserver (observer 1) measurements. Sample size $n=17$ for cTMS and $n=18$ for TT-TMS. SICI measured with the A-SICI protocol (A-SICI, a dimensionless ratio; see Materials and Methods, Statistical analysis, for further explanation) and the T-SICI protocol (T-SICl, in \% RMT). The unit of RMT $0.2 \mathrm{mv}$ and TS Imv $_{1}$ is $\%$ MSO. CR for day 1 (morning and afternoon session 1) and day 2 (morning and afternoon session 2) were calculated to estimate repeatability.

in younger healthy volunteers (Matamala et al., 2018; Samusyte et al., 2018). The repeatability of SICI was improved by averaging multiple ISIs for both techniques, an observation which was also reported in another study (Matamala et al., 2018). This likely reflects the variation (biological and/or technical) of SICI versus ISI curves within subjects, which can be reduced by averaging across ISIs. Nevertheless, averaging is not sufficient to allow a confident use of these measurements for individual decision-making.

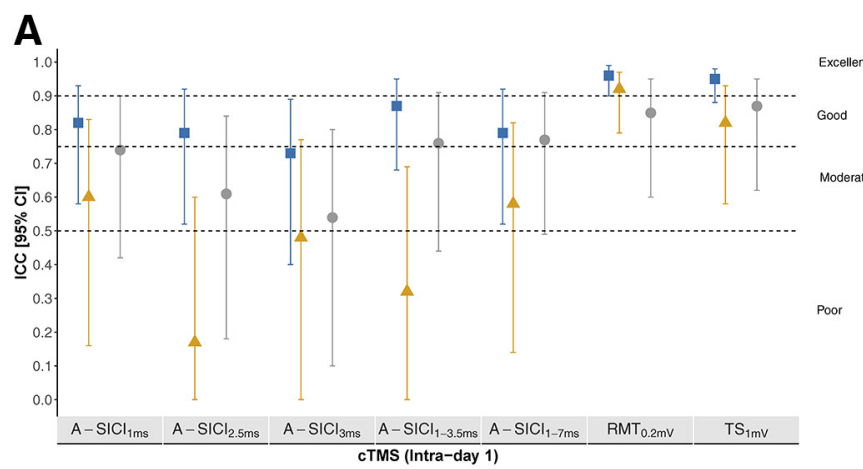

B

C
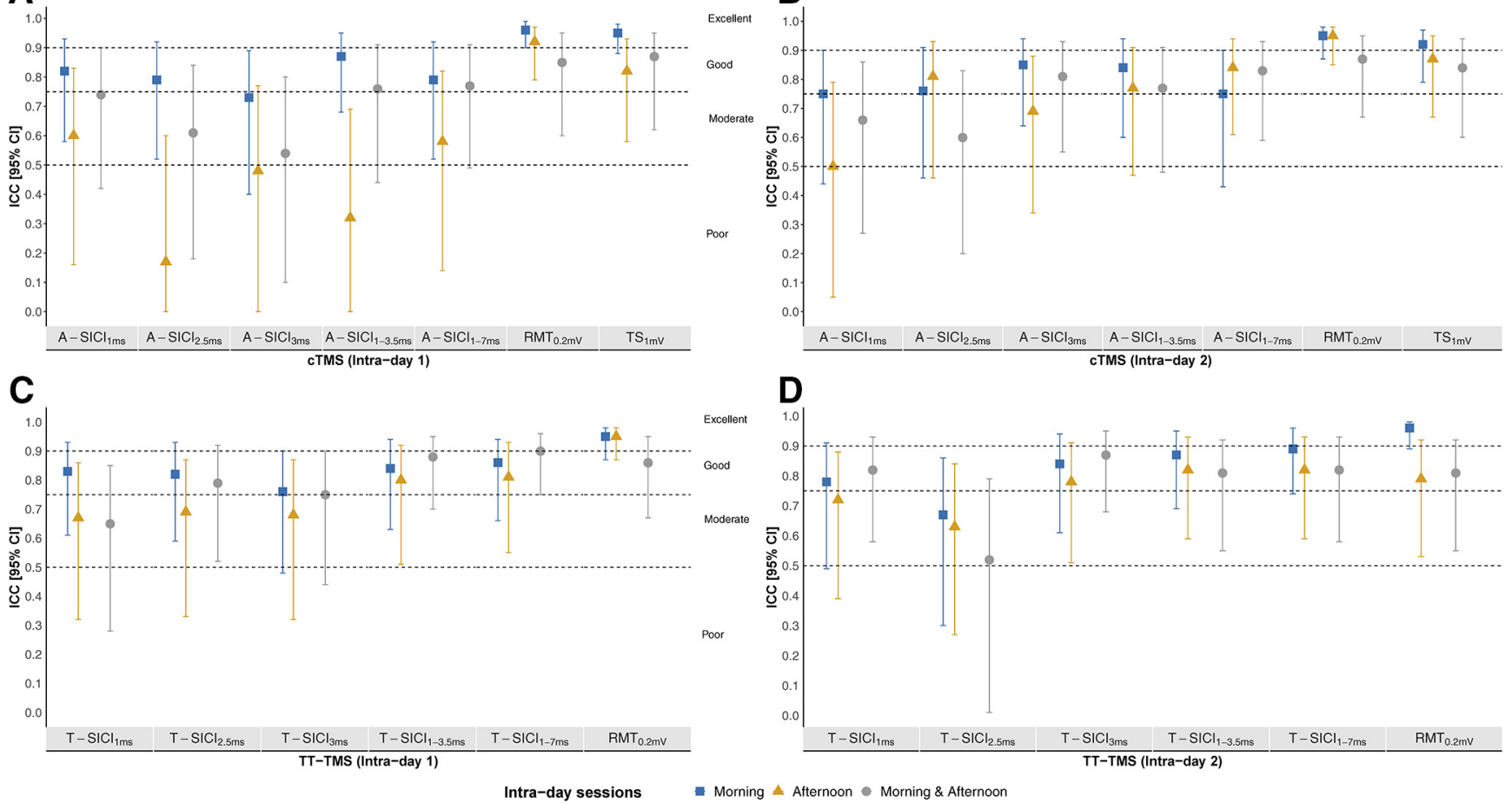

Figure 10. Intraday reliability of intraobserver CTMS and TT-TMS measurements. Intraobserver (observer 1 ) intraday reliability, estimated by ICC, of CTMS $(\boldsymbol{A}, \boldsymbol{B})$ and TT-TMS $(\boldsymbol{C}, \boldsymbol{D})$ parameters from day $1(\boldsymbol{A}-\boldsymbol{C})$ and day $2(\boldsymbol{B}-\boldsymbol{D})$. Sample size was $n=17$ for cTMS and $n=18$ for TT-TMS. $y$-axis: intraday ICC $(2,1)$ with $95 \% \mathrm{Cl}$ for day $1(\boldsymbol{A}-\boldsymbol{C})$ and $2(\boldsymbol{B}-\boldsymbol{D})$. $x$-axis: cTMS and TT-TMS parameters. ICC intervals $<0.5$, between 0.5 and 0.749 , between 0.75 and 0.9 , and intervals $>0.9$ indicated poor, moderate, good, and excellent reliability, respectively. Morning reliability (blue squares), afternoon reliability (yellow triangles), and morning and afternoon reliability (gray circles) are depicted from each measurement day. ICC calculations were based on measurements from the session of interest, i.e., morning session ICCs were based on the two morning measurements. Calculation of "morning and afternoon" reliability was based on data from the first morning measurement and first afternoon measurement. 
A

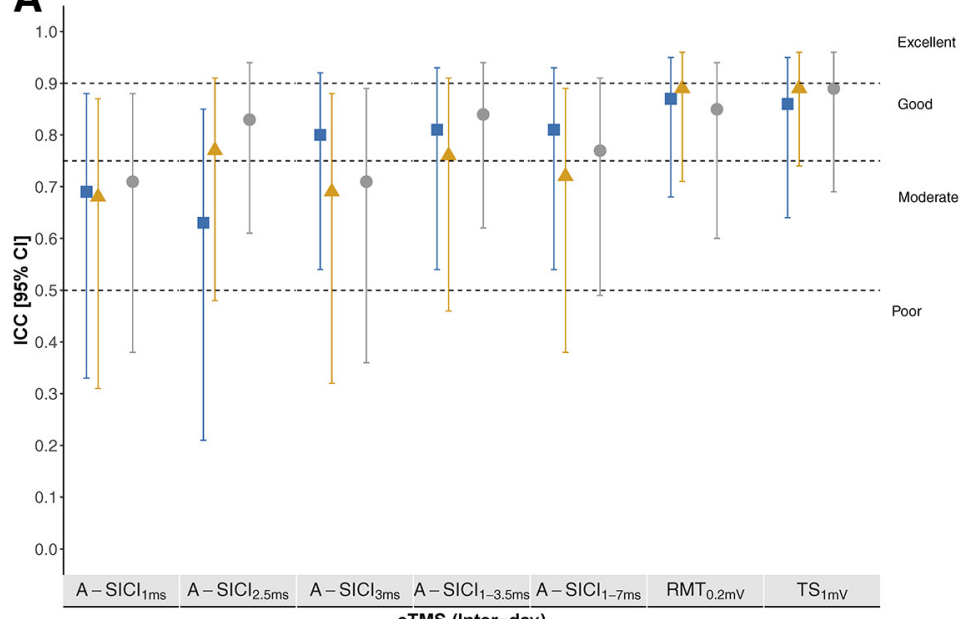

B

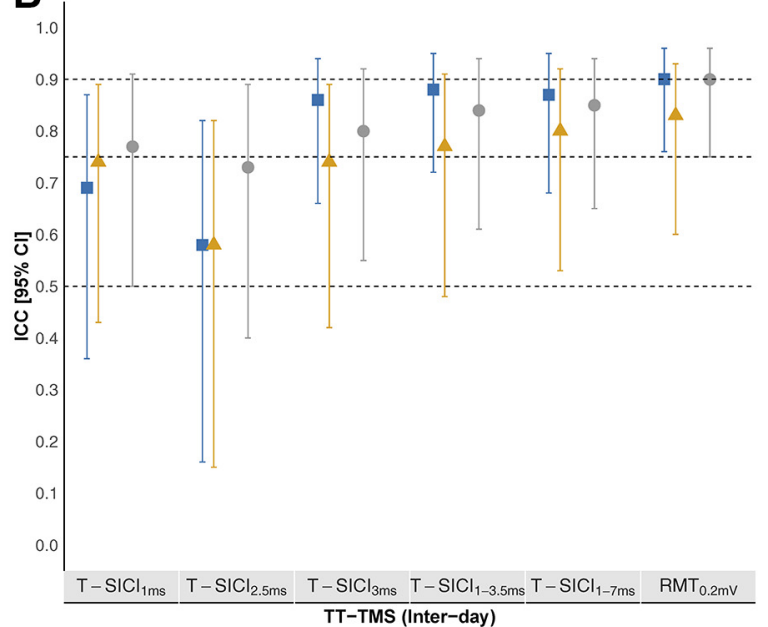

Inter-day sessions Morning $\Delta$ Afternoon - Morning \& Afternoon

Figure 11. Interday reliability of intraobserver cTMS and TT-TMS measurements. Intraobserver (observer 1) interday reliability, estimated by ICC, of CTMS $(\boldsymbol{A})$ and TT-TMS $(\boldsymbol{B})$ parameters. Sample size was $n=17$ for cTMS and $n=18$ for TT-TMS. $y$-axis: interday ICC $(2,1)$ with $95 \%$ CI for CTMS and TT-TMS. $x$-axis: cTMS and TT-TMS parameters. ICC intervals $<0.5$, between 0.5 and 0.749 , between 0.75 and 0.9 , and intervals $>0.9$ indicated poor, moderate, good, and excellent reliability, respectively. Morning reliability (blue squares), afternoon reliability (yellow triangles), and morning and afternoon reliability (gray circles) are depicted. ICC was calculated to estimate interday reliability. Calculation of reliability was done by using data from the first measurement in each session from day 1 and day 2. Calculation of "morning and afternoon" (gray) reliability was based on data from the first morning measurement on day 1 and first afternoon measurement on day 2.

\section{Intraobserver and interobserver reliability}

Overall, $\mathrm{RMT}_{0.2 \mathrm{mv}}$ and $\mathrm{TS}_{1 \mathrm{mv}}$ showed good-to-excellent reliability compared with poor-to-good intraobserver reliability, and poor-to-moderate interobserver reliability of paired-pulse measurements (Figs. 10-12). $\mathrm{SICl}_{3 \mathrm{~ms}}$ and estimates averaged across ISIs tended to have higher interday ICCs, but no consistent pattern was observed for same-day recordings. None of the techniques demonstrated a superior reliability in the present study, in contrast to the previous report (Samusyte et al., 2018). This could be related to methodological differences, but it is also important to note that a direct comparison of ICCs

Table 4. Description of interobserver measurements and reproducibility

\begin{tabular}{|c|c|c|c|c|c|c|c|c|c|}
\hline \multirow[b]{2}{*}{ Method } & \multirow[b]{2}{*}{ Parameter } & \multirow[b]{2}{*}{ Sample size } & \multirow{2}{*}{$\begin{array}{l}\text { Number of } \\
\text { measurements for } \\
\text { each observer }\end{array}$} & \multicolumn{3}{|c|}{ Median [IQR] } & \multicolumn{3}{|c|}{$\begin{array}{c}\text { Student's } \\
\text { paired } \\
t \text { test }\end{array}$} \\
\hline & & & & Observer 1 & & Observer 2 & & $t$ & $p$ value \\
\hline \multirow{5}{*}{ cTMS } & A-SICl $2.5 \mathrm{~ms}$ & 18 & 18 & 25.3 [23.8] & & $40.8[35.1]$ & & 2.11 & 0.0103 \\
\hline & $\mathrm{A}-\mathrm{SICl}_{3 \mathrm{~ms}}$ & 18 & 18 & $43.3[33.1]$ & & $33.7[35.4]$ & & 2.11 & 0.89 \\
\hline & $\mathrm{A}-\mathrm{SICl}_{1-3.5 \mathrm{~ms}}$ & 18 & 108 & $42.2[27.1]^{b}$ & & $31.5[16.5]^{b}$ & & 2.11 & 0.63 \\
\hline & $\mathrm{TS}_{1 \mathrm{mV}}$ & 18 & 18 & 65.9 [12.3] & & $64.5[14.1]$ & & 2.11 & 0.46 \\
\hline & & & & Mean $( \pm S E)^{a}$ & SD & Mean $( \pm S E)^{a}$ & SD & & \\
\hline \multirow[t]{2}{*}{ TT-TMS } & $\mathrm{T}-\mathrm{SICl}_{1 \mathrm{~ms}}$ & 18 & 18 & $9.4( \pm 2.01)$ & 8.9 & $10.5( \pm 1.58)$ & 6.7 & 2.11 & 0.49 \\
\hline & $\mathrm{T}-\mathrm{SICl}_{2.5 \mathrm{~ms}}$ & 18 & 18 & $9.4( \pm 2.49)$ & 10.6 & $6.8( \pm 1.89)$ & 8.0 & 2.11 & 0.28 \\
\hline
\end{tabular}

Reproducibility of interobserver measurements of A-SICI and T-SICI. Sample size $n=18$ for cTMS and TT-TMS for observer 2. Calculation of statistical parameters is based on TMS measurements from observer 2 (from one TMS measurement for each subject) and the corresponding examination (by session, first examination in the session) by observer 1 . A significant difference between observers was seen in $\mathrm{A}-\mathrm{SICl}_{2.5 \mathrm{~ms}}(\mathrm{D}=0.0103)$ only. Differences were interpreted as significant at $p<0.05$.

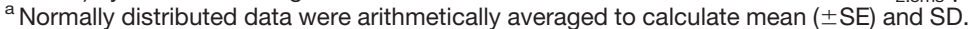

${ }^{b}$ Point estimates for each subject were calculated as geometric means of their measurements. Data are displayed as medians with interquartile ranges [IQR] of subjects' point estimates. 
A

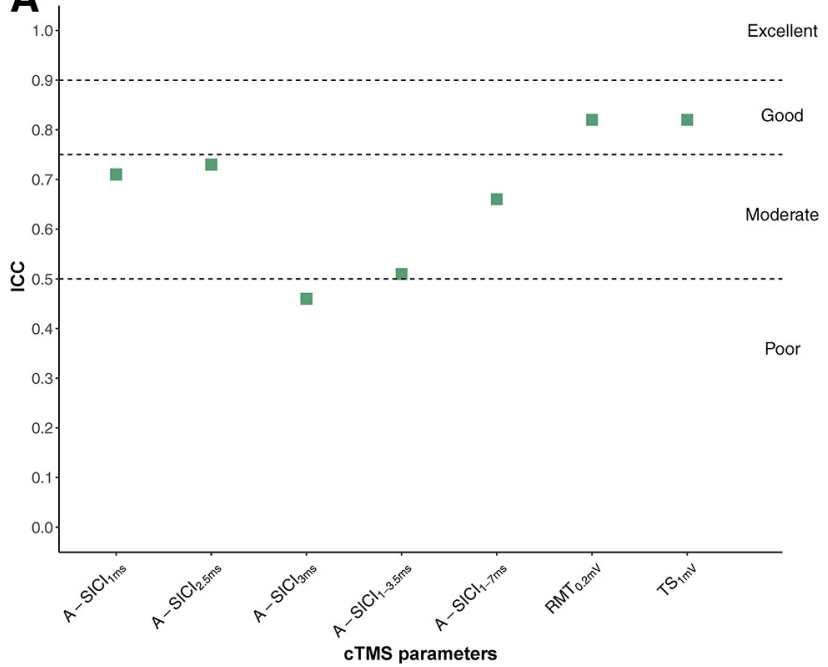

B

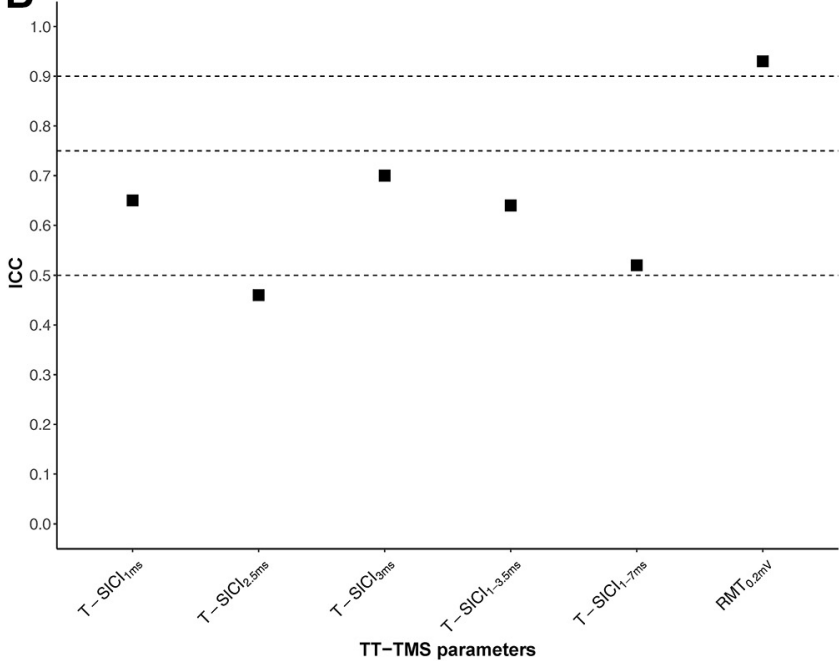

Figure 12. Interobserver reliability of CTMS and TT-TMS measurements. Interobserver (observer 1 and 2)reliability, estimated by ICC, of cTMS $(\boldsymbol{A})$ and TT-TMS $(\boldsymbol{B})$ parameters. Sample size was $n=18 . y$-axis: interobserver ICC $(2,1)$. Calculation of statistical parameters is based on TMS measurements from observer 2 (from one TMS measurement for each subject) and the corresponding examination (by session, first examination in the session) by observer 1. $x$-axis: cTMS (green squares) and TT-TMS (black squares) parameters.

between the studies cannot be made without taking into account the heterogeneity of the samples (Bartlett and Frost, 2008; Streiner and Norman, 2008; Beaulieu et al., 2017).

There was no significant bias in the TMS parameters obtained by different observers and the observer reliability of $\mathrm{RMT}_{0.2 \mathrm{mv}}$ and $\mathrm{TS}_{1 \mathrm{mv}}$ was similar. However, the interobserver ICCs for SICI were generally lower than intraobserver ICCs with both techniques, suggesting that longitudinal measurements should ideally be obtained by the same observer.

\section{Observer and intersession reproducibility}

In general, intraobserver cTMS and TT-TMS measurements were reproducible and no significant differences between examinations were found, except for $\mathrm{A}-\mathrm{SICl}_{2.5 \mathrm{~ms}}$ (between examinations 7 and 8), and two intersessional differences for $\mathrm{T}_{-} \mathrm{SICl}_{3 \mathrm{~ms}}$ (between examinations 4 and 6) and for cTMS TS 1mv $_{1}$ (between examinations 1 and 4). Likewise, no significant interobserver differences were observed, except for the $\mathrm{A}-\mathrm{SICl}_{2.5 \mathrm{~ms}}$ between observer 1 and observer 2 . This suggests that interobserver measurements were reproducible.

As $\mathrm{A}-\mathrm{SICl}_{2.5 \mathrm{~ms}}$ examination 7 by observer 1 was used in both $\mathrm{A}-\mathrm{SICl}$ ratios that turned up statistically significant, it is likely that a bias was introduced in this examination, since it differed both from the same observer's A-SICl${ }_{2.5 m s}$ examination 8 and from observer 2's A-SICl $2.5 \mathrm{~ms}$ examinations. It can only be speculated on, how and why a bias was introduced into only one parameter in just one of the examinations.

Considering the observed statistically significant differences seen with $\mathrm{T}-\mathrm{SICl}_{3 \mathrm{~ms}}$ and with cTMS TS ${ }_{1 \mathrm{mV}}$, it is unclear why exactly these two parameters differ significantly, when none of the other parameters from the same examinations differ. It is possible that even when controlling for familywise error rate (FWER) using the Bonferroni procedure, one or both of the observed statistically significant differences were because of random chance, as a total of four "families" of comparisons were made (Motulsky, 2010).

\section{Is the time of day important?}

There is limited data on the stability of TMS parameters throughout the day. No significant shift in RMT, MEP amplitude or conventional SICI has been previously observed in the awake state during the day (Koski et al., 2005; Lang et al., 2011; ter Braack et al., 2019). Our findings are also consistent with those of a previous study, which found no significant effect of time for SICl when tested at 9 A.M and 4.P.M (Doeltgen and Ridding, 2010). However, it has been proposed that $\mathrm{SICl}$ measurements obtained by TT-TMS may be more reliable if performed in the morning on different days compared with different times on the same day (Matamala et al., 2018). Indeed, it was observed in the present study that most SICI measurements obtained in the morning sessions tended to have better test-retest reliability indices (i.e., higher ICCs and/or lower CRs) compared with the afternoon sessions, both when measured on the same and different experimental days (Figs. 10, 11). Such a pattern was seen with both techniques, though more consistently with TT-TMS. Incidentally, it was observed in the present study that subjects found it more difficult to remain alert during the afternoon sessions. Although recently a nonlinear modulation of corticospinal excitability because of fluctuations in alertness has been described (Noreika et al., 2020), it is unclear whether this could have contributed to the increased variability in the afternoon in the present study. The intraday and interday reliability of $\mathrm{SICl}$ was largely comparable in the present study.

\section{Strengths and limitations}

Automated recording protocols allow the observer to concentrate on coil positioning and minimize observer 
bias, which is crucial for longitudinal assessments and multicentre studies. No considerable systematic bias in the $\mathrm{SICl}$ measurements across multiple ISIs obtained by the same or different observers was found in the present study, supporting the use of such protocols.

The numerous observer examinations are an important strength of the present study as it provides reliability data for different experimental and clinical scenarios: (1) the "immediate" reliability when measurements are repeated in a quick succession (e.g., in studies of interventions with short-lasting effects or a repetition of a test to improve diagnostic certainty in clinics); (2) intraday reliability (e.g., interventional studies in which the effects are measured over the course of the day); (3) interday reliability (e.g., longitudinal assessments in clinical trials).

Although $\mathrm{SICl}$ reliability tended to be better in the morning than in the afternoon sessions, these findings were not statistically significant because of broad and overlapping $95 \% \mathrm{Cl}$. This could be explained by a relatively small sample (Rankin and Stokes, 1998; Bonett, 2002). However, improved precision would require much larger samples (Bonett, 2002), which may not be practical given that ICCs cannot be easily generalized between different samples or populations with different variances (e.g., from healthy volunteers to patients).

While in many fields CRs and ICCs are used to define measurement error, a distinction between technical and biological variability cannot be made for TMS measurements. For example, the increased measurement error in the afternoon may be related to fluctuations in both the subject's state and in the observer's vigilance. Further studies using a robotic arm for coil positioning and thus eliminating the observer factor would shed more light on the biological variability of cortical excitability.

In addition to the different ISIs, SICI ISI averages of 13.5 and 1-7 ms were analyzed for consistency with earlier studies (Matamala et al., 2018; Menon et al., 2018; Ørskov et al., 2021; Tankisi et al., 2021a). Given the interindividual and intraindividual variability of $\mathrm{SICl}$ versus ISI curves, average measures may provide a more reliable parameter. Indeed, SICl averaged across ISIs of 1$7 \mathrm{~ms}$ has shown the best reproducibility (Matamala et al., 2018) and diagnostic utility for ALS in earlier studies with serial tracking (Menon et al., 2015), although some overlap with intracortical facilitation is likely reflected in this measure. Another $\mathrm{SICl}$ variable, an average across ISIs of $1-3.5 \mathrm{~ms}$, has earlier been reported (Ørskov et al., 2021; Tankisi et al., 2021a). It represents intervals with maximum inhibition. However, one should remember that $\mathrm{SICl}$ at different ISIs have different underlying physiological mechanisms related to the refractory period, extrasynaptic and synaptic inhibition and overlap with short interval intracortical facilitation (Ziemann et al., 1996; Peurala et al., 2008; Stagg et al., 2011).

The potential differences as well as advantages and disadvantages of the two techniques have been discussed earlier (Samusyte et al., 2018). Briefly, threshold-tracking may allow a better evaluation of the full inhibitory potential as it overcomes the "floor effect" seen with CTMS. Meanwhile, A-SICI may be more suitable if one is interested in a particular subset of motor neurons. Threshold-tracking protocols can be preferred when the MEP amplitude is low, since in these conditions conventional method will be difficult to perform successfully. In contrast, in subjects with high RMT, the stimulator power may not be sufficient to capture full inhibition with threshold-tracking. As conventional and threshold-tracking protocols may potentially examine different neuron pools in healthy subjects (Samusyte et al., 2018) and patients (Tankisi et al., 2021b), they may have different sensitivity in pathologic conditions or respond differently to drugs. Future head-to-head comparisons in patient populations and interventional studies are warranted.

In conclusion, good correlations between $\mathrm{SICl}$ measurements obtained by cTMS and TT-TMS across a full range of ISIs was observed. The two techniques showed similar test-retest reliability profiles in healthy subjects with poor repeatability on the individual level, and satisfactory reliability on the group level. This suggests that the two automated $\mathrm{SICl}$ protocols may be reliably employed in research studies, but should at this moment be used with caution for individual decision-making in clinical settings. Further studies exploring reliability in different disease cohorts, such as motor neuron diseases or stroke, are warranted to investigate the diagnostic and clinical utility of the two automated $\mathrm{SICl}$ protocols.

\section{References}

Barnhart HX, Barboriak DP (2009) Applications of the repeatability of quantitative imaging biomarkers: a review of statistical analysis of repeat data sets. Transl Oncol 2:231-235.

Bartlett JW, Frost C (2008) Reliability, repeatability and reproducibility: analysis of measurement errors in continuous variables. Ultrasound Obstet Gynecol 31:466-475.

Beaulieu LD, Flamand VH, Massé-Alarie H, Schneider C (2017) Reliability and minimal detectable change of transcranial magnetic stimulation outcomes in healthy adults: a systematic review. Brain Stimul 10:196-213.

Bland JM (2015) Clinical measurement. In: An introduction to medical statistics, Ed 4, pp 315-316. Oxford: Oxford University Press.

Bland JM, Altman D (1986) Statistical methods for assessing agreement between two methods of clinical measurement. Lancet 327:307-310.

Bland JM, Altman DG (1999) Measuring agreement in method comparison studies. Stat Methods Med Res 8:135-160.

Bland JM, Altman DG (2003) Applying the right statistics: analyses of measurement studies. Ultrasound Obstet Gynecol 22:85-93.

Bonett DG (2002) Sample size requirements for estimating intraclass correlations with desired precision. Stat Med 21:1331-1335.

Brown KE, Lohse KR, Mayer IMS, Strigaro G, Desikan M, Casula EP, Meunier S, Popa T, Lamy JC, Odish O, Leavitt BR, Durr A, Roos RAC, Tabrizi SJ, Rothwell JC, Boyd LA, Orth M (2017) The reliability of commonly used electrophysiology measures. Brain Stimul 10:1102-1111.

De Goede AA, Van Putten MJAM (2019) Infraslow activity as a potential modulator of corticomotor excitability. J Neurophysiol 122:325-335.

de Vet HCW, Terwee CB, Knol DL, Bouter LM (2006) When to use agreement versus reliability measures. J Clin Epidemiol 59:10331039.

Doeltgen SH, Ridding MC (2010) Behavioural exposure and sleep do not modify corticospinal and intracortical excitability in the human motor system. Clin Neurophysiol 121:448-452. 
Fisher RJ, Nakamura Y, Bestmann S, Rothwell JC, Bostock H (2002) Two phases of intracortical inhibition revealed by transcranial magnetic threshold tracking. Exp Brain Res 143:240-248.

Fleiss JL (1999) Reliability of measurement. In: The design and analysis of clinical experiments, pp 1-32. Hoboken: Wiley, Inc.

Fleming MK, Sorinola IO, Newham DJ, Roberts-Lewis SF, Bergmann JHM (2012) The effect of coil type and navigation on the reliability of transcranial magnetic stimulation. IEEE Trans Neural Syst Rehabil Eng 20:617-625.

Goetz SM, Luber B, Lisanby SH, Peterchev AV (2014) A novel model incorporating two variability sources for describing motor evoked potentials. Brain Stimul 7:541-552.

Groppa S, Oliviero A, Eisen A, Quartarone A, Cohen LG, Mall V, Kaelin-Lang A, Mima T, Rossi S, Thickbroom GW, Rossini PM, Ziemann U, Valls-Solé J, Siebner HR (2012) A practical guide to diagnostic transcranial magnetic stimulation: report of an IFCN committee. Clin Neurophysiol 123:858-882.

Kiers L, Cros D, Chiappa KH, Fang J (1993) Variability of motor potentials evoked by transcranial magnetic stimulation. Electroencephalogr Clin Neurophysiol 89:415-423.

Koo TK, Li MY (2016) A guideline of selecting and reporting intraclass correlation coefficients for reliability research. J Chiropr Med 15:155-163.

Koski L, Schrader LM, Wu AD, Stern JM (2005) Normative data on changes in transcranial magnetic stimulation measures over a ten hour period. Clin Neurophysiol 116:2099-2109.

Kujirai T, Caramia MD, Rothwell JC, Day BL, Thompson PD, Ferbert A, Wroe S, Asselman P, Marsden CD (1993) Corticocortical inhibition in human motor cortex. J Physiol 471:501-519.

Lang N, Rothkegel H, Reiber H, Hasan A, Sueske E, Tergau F, Ehrenreich H, Wuttke W, Paulus W (2011) Circadian modulation of GABA-mediated cortical inhibition. Cereb Cortex 21:2299-2306.

Matamala JM, Howells J, Dharmadasa T, Trinh T, Ma Y, Lera L, Vucic S, Burke D, Kiernan MC (2018) Inter-session reliability of short-interval intracortical inhibition measured by threshold tracking TMS. Neurosci Lett 674:18-23.

Menon P, Geevasinga N, Yiannikas C, Howells J, Kiernan MC, Vucic $S$ (2015) Sensitivity and specificity of threshold tracking transcranial magnetic stimulation for diagnosis of amyotrophic lateral sclerosis: a prospective study. Lancet Neurol 14:478-484.

Menon P, Kiernan MC, Vucic S (2018) Cortical excitability varies across different muscles. J Neurophysiol 120:1397-1403.

Motulsky H (2010) Intuitive biostatistics: a nonmathematical guide to statistical thinking, Ed 2. New York: Oxford University Press.

Ngomo S, Leonard G, Moffet H, Mercier C (2012) Comparison of transcranial magnetic stimulation measures obtained at rest and under active conditions and their reliability. J Neurosci Methods 205:65-71.

Nielsen JF (1996) Logarithmic distribution of amplitudes of compound muscle action potentials evoked by transcranial magnetic stimulation. J Clin Neurophysiol 13:423-434.

Noreika V, Kamke MR, Canales-Johnson A, Chennu S, Bekinschtein TA, Mattingley JB (2020) Alertness fluctuations when performing a task modulate cortical evoked responses to transcranial magnetic stimulation. Neuroimage 223:117305.

Ørskov S, Bostock H, Howells J, Pugdahl K, Fuglsang-Frederiksen A, Nielsen CSZ, Cengiz B, Samusyte G, Koltzenburg M, Tankisi H (2021) Comparison of figure-of- 8 and circular coils for threshold tracking transcranial magnetic stimulation measurements. Neurophysiol Clin $51: 153-160$

Peurala SH, Müller-Dahlhaus JF, Arai N, Ziemann U (2008) Interference of short-interval intracortical inhibition $(\mathrm{SICl})$ and short-interval intracortical facilitation (SICF). Clin Neurophysiol 119:2291-2297.
Rankin G, Stokes M (1998) Reliability of assessment tools in rehabilitation: an illustration of appropriate statistical analyses. Clin Rehabil 12:187-199.

Roshan L, Paradiso GO, Chen R (2003) Two phases of short-interval intracortical inhibition. Exp Brain Res 151:330-337.

Rossi S, Hallett M, Rossini PM, Pascual-Leone A (2011) Screening questionnaire before TMS: an update. Clin Neurophysiol 122:1686.

Rossini PM, Burke D, Chen R, Cohen LG, Daskalakis Z, Di lorio R, Di Lazzaro V, Ferreri F, Fitzgerald PB, George MS, Hallett M, Lefaucheur JP, Langguth B, Matsumoto H, Miniussi C, Nitsche MA, Pascual-Leone A, Paulus W, Rossi S, Rothwell JC, et al. (2015) Non-invasive electrical and magnetic stimulation of the brain, spinal cord, roots and peripheral nerves: basic principles and procedures for routine clinical and research application: an updated report from an I.F.C.N. committee. Clin Neurophysiol 126:1071-1107.

Samusyte G, Bostock H, Rothwell J, Koltzenburg M (2018) Short-interval intracortical inhibition: comparison between conventional and threshold-tracking techniques. Brain Stimul 11:806-817.

Schambra HM, Ogden RT, Martínez-Hernández IE, Lin X, Chang YB, Rahman A, Edwards DJ, Krakauer JW (2015) The reliability of repeated TMS measures in older adults and in patients with subacute and chronic stroke. Front Cell Neurosci 9:335.

Stagg CJ, Bestmann S, Constantinescu AO, Moreno Moreno L, Allman C, Mekle R, Woolrich M, Near J, Johansen-Berg H, Rothwell JC (2011) Relationship between physiological measures of excitability and levels of glutamate and GABA in the human motor cortex. J Physiol 589:5845-5855.

Streiner DL, Norman GR (2008) Health measurement scales: a practical guide to their development and use. New York: Oxford University Press.

Tankisi H, Cengiz B, Howells J, Samusyte G, Koltzenburg M, Bostock H (2021a) Short-interval intracortical inhibition as a function of inter-stimulus interval: three methods compared. Brain Stimul 14:22-32.

Tankisi H, Nielsen CS-Z, Howells J, Cengiz B, Samusyte G, Koltzenburg M, Blicher JU, Møller AT, Pugdahl K, FuglsangFrederiksen A, de Carvalho M, Bostock H (2021b) Early diagnosis of amyotrophic lateral sclerosis by threshold tracking and conventional transcranial magnetic stimulation. Eur J Neurol 28: 30303039.

ter Braack EM, de Goede AA, van Putten MJAM (2019) Resting motor threshold, MEP and TEP variability during daytime. Brain Topogr 32:17-27.

Vucic S, Kiernan MC (2006) Novel threshold tracking techniques suggest that cortical hyperexcitability is an early feature of motor neuron disease. Brain 129:2436-2446.

Vucic S, Kiernan MC (2008) Cortical excitability testing distinguishes Kennedy's disease from amyotrophic lateral sclerosis. Clin Neurophysiol 119:1088-1096.

Vucic S, Rutkove SB (2018) Neurophysiological biomarkers in amyotrophic lateral sclerosis. Curr Opin Neurol 31:640-647.

Vucic S, Howells J, Trevillion L, Kiernan MC (2006) Assessment of cortical excitability using threshold tracking techniques. Muscle Nerve 33:477-486.

Vucic S, Cheah BC, Krishnan AV, Burke D, Kiernan MC (2009) The effects of alterations in conditioning stimulus intensity on short interval intracortical inhibition. Brain Res 1273:39-47.

Ziemann U, Lönnecker S, Steinhoff BJ, Paulus W (1996) The effect of lorazepam on the motor cortical excitability in man. Exp Brain Res 109:127-135

Zrenner C, Desideri D, Belardinelli P, Ziemann U (2018) Real-time EEG-defined excitability states determine efficacy of TMS-induced plasticity in human motor cortex. Brain Stimul 11:374-389. 